\title{
Novel and Powerful 3D Adaptive Crisp Active Contour Method applied in the Segmentation of CT Lung Images
}

\author{
Pedro Pedrosa Rebouças Filho ${ }^{\mathrm{a}}$, Paulo César Cortez ${ }^{\mathrm{b}}$, Antônio C. da Silva \\ Barros $^{c}$, Victor Hugo C. Albuquerque ${ }^{\mathrm{c}}$, João Manuel R. S. Tavares ${ }^{\mathrm{d}}$ \\ ${ }^{a}$ Laboratório de Processamento de Imagens e Simulação Computacional, Instituto \\ Federal de Educação, Ciência e Tecnologia do Ceará, Maracanau, CE, Brazil \\ ${ }^{b}$ Departamento de Engenharia de Teleinformática, Universidade Federal do Ceará, \\ Fortaleza, CE, Brazil \\ ${ }^{c}$ Programa de Pós-Graduação em Informática Aplicada, Laboratório de Bioinformática, \\ Universidade de Fortaleza, Fortaleza, Ceará, Brazil \\ ${ }^{d}$ Instituto de Ciência e Inovação em Engenharia Mecânica e Engenharia Industrial, \\ Departamento de Engenharia Mecânica, Faculdade de Engenharia, Universidade do \\ Porto, Porto, Portugal
}

\begin{abstract}
The World Health Organization estimates that 300 million people have asthma, 210 million people have Chronic Obstructive Pulmonary Disease (COPD), and, according to WHO, COPD will become the third major cause of death worldwide in 2030. Computational Vision systems are commonly used in pulmonology to address the task of image segmentation, which is essential for accurate medical diagnoses. Segmentation defines the regions of the lungs in CT images of the thorax that must be further analyzed by the system or by a specialist physician. This work proposes a novel and powerful technique named 3D Adaptive Crisp Active Contour Method (3D ACACM) for the segmentation of CT lung images. The method starts with a sphere within the lung to be segmented that is deformed by forces acting on it towards the lung borders. This process is performed iteratively in order to minimize an energy function associated with the 3D deformable model used. In the exper-

\footnotetext{
${ }^{*}$ Corresponding author's email: tavares@fe.up.pt

Email addresses: pedrosarf@ifce.edu.br (Pedro Pedrosa Rebouças Filho), cortez@lesc.ufc.br (Paulo César Cortez), carlosbarros@lapisco.ifce.edu.br (Antônio C. da Silva Barros), victor .albuquerque@unifor.br (Victor Hugo C. Albuquerque), tavares@fe.up.pt (João Manuel R. S. Tavares)
} 
imental assessment, the 3D ACACM is compared against three approaches commonly used in this field: the automatic 3D Region Growing, the level-set algorithm based on coherent propagation and the semi-automatic segmentation by an expert using the 3D OsiriX toolbox. When applied to $40 \mathrm{CT}$ scans of the chest the 3D ACACM had an average F-measure of $99.22 \%$, revealing its superiority and competency to segment lungs in CT images.

Keywords: CT scan; Image Segmentation; 3D Reconstruction; Lung Structures.

\section{Introduction}

Several diseases that affect the world population are related to the lungs, for example: asthma (Kwan et al., 2015); Wisniewski and Zielinskil, 2015), bronchiectasis (Arunkumar, 2012) and chronic obstructive pulmonary disease (COPD) (Mieloszyk et al., 2014; Ramalho et al., 2014; Spina et al., 2015).

The World Health Organization (WHO) estimates that 300 million people have asthma, and this disease causes about 250 thousand deaths per year worldwide (Campos and Lemos, 2010). Also, 210 million people have COPD and more than 300 thousand people died in 2005 from this disease (WH(), 2014). Recent studies have shown that COPD is present in the 20 to 45 yearold-age bracket, although people over 50 years old are the most commonly affected. Additionally, WHO estimates that COPD will be the third major cause of death worldwide by 2030 (Marco et al., 20(1) ). For example, in Brazil from 1992 to 2006, 15\% of all hospital admissions financed by the national public health system were due to respiratory diseases, and asthma and COPD together were responsible for 562,016 cases (Campos and Lemos, 20(0). $)$.

Hence, early effective diagnosis of lung diseases is urgently needed in public health. Among the factors that contribute to achieve this goal is the increased accuracy of the diagnoses made by specialized physicians with the aid of computational vision systems. Additionally, some computational techniques can monitor patients with asthma and COPD using personal devices. Examples that can be highlighted among these techniques are the works of Kwan et al. (2015) and Juen et al. (2015).

In pulmonology, computed tomography (CT) imaging is often used as a tool for detection and monitoring of diseases. Hence, CT images have been used in the analysis of airways (Pu et al, 2017; Lo et al, 2012), vessels 
(Korfiatis et al., 201]), cancer nodules (Diciotti et al., 201]), pulmonary lobes (Van Rikxoort et al., 2010), pulmonary emphysema (Sorensen et all, 2012; Hame et al., 2014), and fibrosis (Ariani et al., 2014) among other lung diseases. Additionally, computational vision systems have been used as diagnostic tools, particularly to address image segmentation, which is an essential step to assure correct and accurate results, by identifying the region of the lungs in the CT thorax images that must be further analyzed by the system or by specialists.

The segmentation of objects or structures in medical images is usually more complex than in other types of images. Furthermore, in the case of lung images, this difficulty is due to the variability of the structures and the internal organs of the lungs that can be imaged from different planes. Also, diseases can affect these organs, increasing the difficulty even more to develop effective techniques to segment the images under study (Rebouças Filho et al., 2010, 2013).

Various lung segmentation techniques have been developed in recent years. Among these techniques, the 3D Region Growing (3D RG) approach has been applied to segment the lung and related internal structures, such as the vessels and airways (Born et al, 201)9; Irving et al., 201).9; Tschirren et al., 201(1); Matsuoka et al., 2010; De Nunzio et al., 2017). Commercial software packages commonly combine the 3D RG approach and Human Anatomy information, like HU density ranges, to aid image-based medical diagnoses. However, a correct analysis is more difficult when there is a disease in the lungs. The work of Nemec et al. (2015) studied and evaluated four software packages commonly used to extract the lung volume of healthy volunteers from CT images of the chest.

Among the softwares available, OsiriX from the University of Geneva (http://osirix-viewer.com//) is widely used for viewing and rendering 3D medical images (Canas et al, 2007; Martin et al, 2013; Wink, 2014). This software has automatic and semi-automatic tools for 3D segmentation (Michael P (hae, 2015); Presti et al, 2015). In semi-automatic segmentation, an expert analyzes the 3D objects under analysis and removes unwanted objects using the 3D Toolbox (Michael P (hae, 2015).

Wang et al. (201), 2014) developed a fast level-set algorithm based on the coherent propagation method and assessed its use on clinical datasets. The results indicated that this algorithm was about 10 times faster than the ITK Snap software in the segmentation of medical images.

Mansoor et al. (2014) presented a solution to segment healthy and dis- 
eased lungs in 3D using fuzzy logic and texture. On other hand, Wei and Lil (2014) presented a 3D lung segmentation solution based on machine learning techniques, obtaining an accuracy difference of $2 \%$ relatively to experts.

Sun et al. (2012) proposed the Robust Active Shape Model approach for the segmentation of lungs showing regions with cancer. The evaluation of the approach was very limited; however, it demonstrated that active contours can be effectively used for this purpose.

The method proposed in this work is a new Active Contour Model called 3D Adaptive Crisp Active Contour Method (3D ACACM). The proposed method aims to increase the accuracy and reduce the analysis time and subjectivity in the segmentation and analysis of CT scans of the chest by specialized physicians. The method has the advantages of the works from Mansoor et al. (2014), Wei and Lil (2014) and Sun et al. (2012), and combines machine learning techniques with active contours in order to segment lungs efficiently in 3D .

Active Contour Models (ACMs) can be divided into parametric and geometric models. Parametric ACMs move the segmentation curve by minimizing the energy required based on its shape and image information (Moallem et al, 2015; Ge et al, 2016; Moreira et al, 2016). There are several 2D versions of parametric ACMs in the literature, which are commonly known as Snakes. On the other hand, geometric ACMs move the curve by minimizing the energy required based on a function of statistical probability (Leninisha and Vanil, 2015; Mesejo et al., 2015; Rebouças et al., 2016). There are different versions of these models in 2D and 3D, which are commonly called Level Set models, including the Geodesic model (Dicintti et al, 2017; Qiu et al., 2015) and other models developed to optimize performance (Wang et al., [2017, 2014).

This paper proposes a new parametric 3D active contour model specifically to segment complex objects such as the lung, and not only objects with cylindrical topology and regular shape, as the one proposed in Schmitter et al (2015). The proposed method is innovative in terms of 3D segmentation, because the points of the 3D model are moved using information based on the 3D shape of the model and image voxel information, which is different compared to the existing 3D ACMs for complex shapes based on geometric modeling. The results show the gain in terms of computation time and accuracy against to the related $2 \mathrm{D}$ version due to the new formulation used, and its superiority in comparison to other 3D methods that are commonly used for the same purpose. 
In the experimental assessment, the 3D ACACM is compared against three methods commonly used in this field: the automatic 3D Region Growing (3D RG), the level-set algorithm based on the coherent propagation method (LSCPM) and the semi-automatic segmentation by an expert using the 3D OsiriX. All methods are compared in terms of F-measure and processing time to segment lungs in 3D CT images of the thorax.

\section{Proposed method}

In this section, a new 3D segmentation method based on the principles of Active Contour Models, called 3D Adaptive Crisp ACM, is described. All the steps of the new 3D method proposed, using information from 3D medical images, are described in this section from the initialization to the stabilization of the segmentation model.

Unlike other parametric ACMs, the proposed method moves the points of the model using information from image voxels and model shape. Thus, one point $m(s)$ is moved by minimizing the energy of the 3D Adaptive Crisp $\mathrm{ACM} E_{C A_{3 D}}$, which is given by:

$$
\begin{aligned}
& E_{C A_{3 D}}[m(s)]= \\
& E_{\text {int }_{\text {ddap }_{3 D}}}[m(s)]+\tau E_{\text {ext }_{A C E E_{3 D}}}[m(s)],
\end{aligned}
$$

where $E_{\text {int }_{a d a p}{ }_{3 D}}[m(s)]$ is the 3D Adaptive Internal Energy and $E_{\text {ext }_{A C E E_{3 D}}}[m(s)]$ is the 3D Adaptive Crisp External Energy, which are both proposed in this work. A point $m$ of the 3D model has as coordinate a $C$ curve in a slice $i$ of the axis $z$. Thus, $\left.m(s)=\left[c(s), z_{i}\right)\right]$, where $c(s)$ is composed of the $[x(s), y(s)]$ coordinates, and $z_{i}$ is the plane of the curve $c$. The position of point $c(s)$ is on the axis $z$.

As aforementioned, the proposed method follows the concept of the 2D method presented in Rebouças Filho et al. (2013). However, the energies of the new 3D model were reformulated to increase the segmentation speed and the stability. This the first parametric ACM proposed to efficiently segment complex objects in 3D. 


\subsection{D Adaptive Internal Energy}

The internal energy of the proposed 3D parametric ACM is calculated based on 3D model information:

$$
\begin{gathered}
E_{\text {int }_{a d a p_{3 D}}}[m(s)]= \\
\beta F_{\text {cont }_{3 D}}[m(s)]+\alpha F_{\text {adap }_{3 D}}[m(s)],
\end{gathered}
$$

where $F_{\text {cont }_{3 D}}[m(s)]$ is called 3D Continuity Force, $F_{\text {adap }}{ }_{3 D}[m(s)]$ is the Adaptive Force, and $\beta$ and $\alpha$ are weights to set the importance of these forces in the final internal energy of the model $E_{\text {int }_{\text {adap }} 3 \mathrm{D}}$.

\subsubsection{D Continuity Force}

The reformulation of this energy in the proposed method aimed to keep the points of the 3D model equidistant considering not only the neighboring points in the same slice, but also maintaining the distance between the points of the neighboring slices. Therefore, by increasing the distance of the closest points and reducing the distance of the furthest ones, the 3D continuity force tends to increase the stability of the model.

The calculation of the 3D Continuity Force $F_{\text {cont }_{3 D}}$ is performed by using a distance between two 3D points using the coordinates $x, y$ and $z$, given by:

$$
d_{3 D}=\sqrt{\Delta x^{2}+\Delta y^{2}+\Delta z^{2}},
$$

where $\Delta x, \Delta y$ and $\Delta z$ correspond to the differences of the point coordinates on the axes $x, y$ and $z$, respectively, which leads to:

$$
\begin{gathered}
F_{\text {cont }_{3 D}}\left[x(s), y(s), z_{i}\right]=F_{\text {cont }_{3 D z_{i}}}\left[x(s), y(s), z_{i}\right]+ \\
F_{\text {cont }_{3 D z_{i-1}}}\left[x(s), y(s), z_{i}\right]+F_{\text {cont }_{3 D z_{i+1}}}\left[x(s), y(s), z_{i}\right],
\end{gathered}
$$

where $F_{\text {cont }_{3 D z_{i}}}, F_{\text {cont }_{3 D z_{i-1}}}$ and $F_{\text {cont }_{3 D z_{i+1}}}$ are obtained from regions of the slices $i, i-1$ and $i+1$, respectively, and:

$$
\begin{gathered}
F_{\text {cont }_{3 D_{z_{i}}}}\left[x(s), y(s), z_{i}\right]= \\
\left|A D-\sqrt{\left[x(s)_{z_{i}}-x(s-1)_{z_{i}}\right]^{2}+\left[y(s)_{z_{i}}-y(s-1)_{z_{i}}\right]^{2}}\right| \\
+\left|A D-\sqrt{\left[x(s)_{z_{i}}-x(s+1)_{z_{i}}\right]^{2}+\left[y(s)_{z_{i}}-y(s+1)_{z_{i}}\right]^{2}}\right|,
\end{gathered}
$$




$$
\begin{gathered}
F_{\text {cont }_{3 D z_{i-1}}}\left[x(s), y(s), z_{i}\right]= \\
\left|A D-\sqrt{\left[x(s)_{z_{i}}-x_{p_{z_{i-1}}}\right]^{2}+\left[y(s)_{z}-y_{p_{z_{i-1}}}\right]^{2}+d_{z}^{2}}\right|,
\end{gathered}
$$

and

$$
\begin{gathered}
F_{\text {cont }_{3 D z_{i+1}}}\left[x(s), y(s), z_{i}\right]= \\
\left|A D-\sqrt{\left[x(s)_{z_{i}}-x_{p_{z_{i+1}}}\right]^{2}+\left[y_{z}(s)-y_{p_{z_{i+1}}}\right]^{2}+d_{z}^{2}}\right| .
\end{gathered}
$$

In Eqs. 15, [ and 7, $A D$ is the average distance among the 3D model points, $\left[x(s), y(s), z_{i}\right]$ are the coordinates of point $[x(s), y(s)]$ of the $z_{i}$ curve in the slice where the force is calculated. The $\left[x_{p_{z_{i-1}}}, y_{p_{z_{i-1}}}\right]$ and $\left[x_{p_{z_{i+1}}}, y_{p_{z_{i+1}}}\right]$ points are the $[x(s), y(s)]$ in $i-1$ and $i+1$ slices, respectively, and $d_{z}$ is the distance between the curves in different slices in the $z$ axis, which is constant for each dataset. Note that $[x(s-1), y(s-1)]$ and $[x(s+1), y(s+1)]$ are neighbors of point $[x(s), y(s)]$ in the $z_{i}$ slice; therefore, $F_{\text {cont }_{3 D z_{i}}}$ does not have $d z$ in the calculation.

Figure $\square$ shows an example of the points and the distances involved in the calculation of the force $F_{a d a p_{3 D}}$ described by Eq. 目 taking a point $C_{i}$ as a reference. This figure illustrates the distances used in Eq. 5 in green (slice i), and the ones in Eqs. [6 and $\square$ in red (slices $i-1$ and $i+1$, respectively).

The resultant force $F_{\text {cont }_{3 D}}$ uses the average distance between the points in the model $(A D)$. This parameter is used as a target of the analyzed distances, generating forces that increase the distances that are inferior to $A D$ and reduce the distances that are superior to $A D$. Thus, the $3 \mathrm{D}$ continuity model force tends to make the connections between the model points equally spaced in $3 \mathrm{D}$. The average distance $A D$ needs to be updated at each iteration, because when the points of the 3D model are moved, the distances between them change. As result, this energy prevents that the points of the model from moving uncoordinatedly not only in relation to the neighboring points in the same slice, but also in relation to the neighboring points in the slices above and below (Figure $\mathbf{T}$ ).

This strategy tends to improve the stability of the model.

\subsubsection{D Adaptive Balloon Force}

In the proposed method, the reformulation of this energy to 3D aimed to keep the scope of the segmentation in different directions, but with an even 


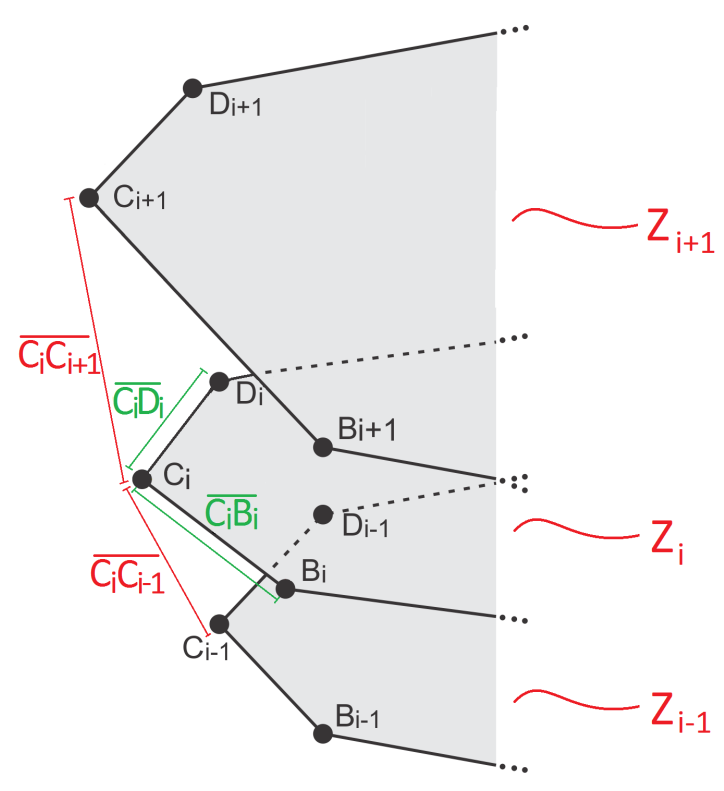

Figure 1: Illustration of the distances used to calculate the 3D Continuity Force, with the distances used in Eq. [ in green (slice i), and the distances used in Eqs. [6 and $\square$ in red (slices $i-1$ and $i+1$, respectively).

faster rate than in the 2D method (Rebouças Filho et al., 2013; Rebouças Filho et al, 2014). This is possible by using information from neighboring slices to boost the movement of the model points.

The 3D Adaptive Balloon Force proposed in this work uses the topology of each point to move it, and takes into account the information of neighboring slices in the calculation of this force that will expand the model to 3D. Thus, this force must use the topology of 3 slices to move each point, increasing the convergence of each point towards the object of interest. The quality of information on the object of interest improves when the proposed model uses three consecutive slices into account, $i, i-1$ and $i+1$, where $i$ is the slice of the point being analyzed.

Thus, the 3D Adaptive Balloon Force $F_{a d a p_{3 D}}$ at a given point $[c(s)]$ belonging to the slice $z_{i}$, whose coordinates are $\left[x(s)_{z_{i}}, y(s)_{z_{i}}\right]$, is given by:

$$
\begin{gathered}
F_{\text {adap } 3 D_{3}}\left[c(s), z_{i}\right]=F_{\text {adap }_{3 D z_{i}}}\left[c(s), z_{i}\right]+ \\
F_{\operatorname{adap}_{3 D z_{i-1}}}\left[c(s), z_{i}\right]+F_{a d a p_{3 D z_{i+1}}}\left[c(s), z_{i}\right],
\end{gathered}
$$


where $F_{a d a p_{3 D z_{i}}}, F_{a d a p_{3 D z_{i-1}}}$ and $F_{a d a p_{3 D z_{i+1}}}$ use the nearest point of $c(s)$ in the curves from slices $i, i-1$ and $i+1$, respectively, and are defined as:

$$
\begin{gathered}
F_{\text {adap }_{3 D z_{i}}}\left[c(s), z_{i}\right]= \\
\sqrt{\left|x(s)_{z_{i}} \pm x_{m_{z_{i}}}\right|^{2}+\left|y(s)_{z_{i}} \pm y_{m_{z_{i}}}\right|^{2}} \\
F_{\text {adap }_{3 D z_{i-1}}}\left[c(s), z_{i}\right]= \\
\sqrt{\mid x(s)_{z_{i}} \pm x_{\left.p_{z_{i-1}}\right|^{2}+\left|y(s)_{z_{i}} \pm y_{p_{z_{i-1}}}\right|^{2}}}
\end{gathered}
$$

and

$$
\begin{gathered}
F_{\text {adap } 3 p_{z+1}}\left[c(s), z_{i}\right]= \\
\sqrt{\left|x(s)_{z_{i}} \pm x_{p_{z_{i+1}}}\right|^{2}+\left|y(s)_{z_{i}} \pm y_{p_{z_{i+1}}}\right|^{2}}
\end{gathered}
$$

where point $\left[x_{m_{z_{i}}}, y_{m_{z_{i}}}\right]$ is the center point of the neighboring of point $c(s)$ from the curve in slice $i$ of $z$ axis as they are in the same slice, while points $\left[x_{p_{z_{i-1}}}, y_{p_{z_{i-1}}}\right]$ and $\left[x_{p_{z_{i+1}}}, y_{p_{z_{i+1}}}\right]$ are nearest to curve in slice $i-1$ and $i+1$ of the $z$ axis, respectively. Points $\left[x_{p_{z_{i-1}}}, y_{p_{z_{i-1}}}\right]$ and $\left[x_{p_{z_{i+1}}}, y_{p_{z_{i+1}}}\right]$ are the same as those used in the calculation of the 3D Continuity Force described

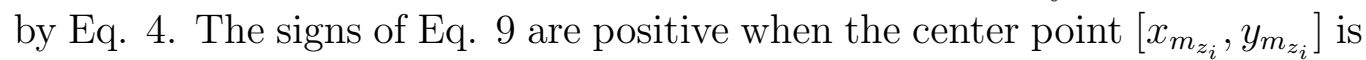
internal to curve $c$ of slice $z$, and negative otherwise. On the other hand, the signs of Eqs. [0] and प] are defined by points $\left[x_{p_{z_{i-1}}}, y_{p_{z_{i-1}}}\right]$ and $\left[x_{p_{z_{i+1}}}, y_{p_{z_{i+1}}}\right]$, respectively. Therefore, the signs are positive when these points are internal to curve $c$ of slice $i$ and negative otherwise.

The 3D Adaptive Force expands the model based on two pieces of information to calculate the energy of each point. The first is determined by the nearest points of the model when it comes to the neighboring slices, $i-1$ and $i+1$, and is determined by the center point of the neighbors when it comes to the same slice $i$ of axis $z$. These points are analyzed by extruding or attracting the point according to their self-analysis, using the solution suggested by Berg et al. (1475), which defines if the point is inside or outside the model.

An example of the performance of the components of force $F_{a d a p_{3 D}}$ acting on a point $C_{i}$, as described in Eq. 8, is shown in Figure 2. In this figure, the 


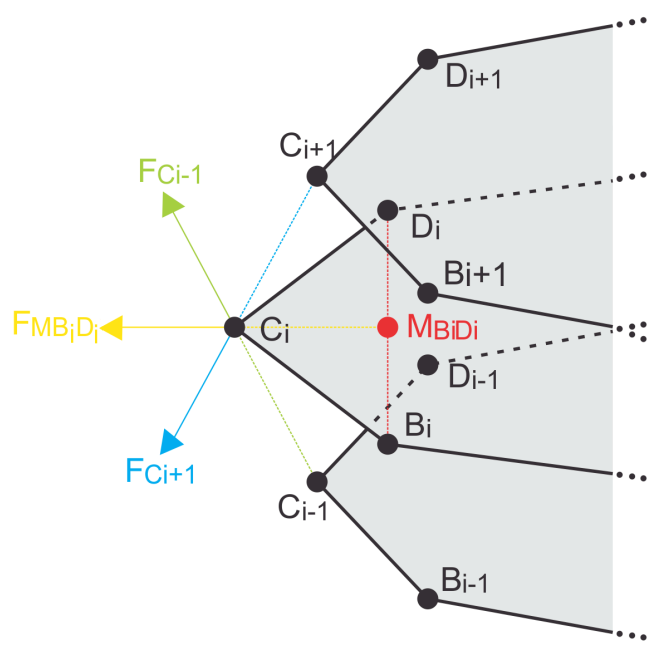

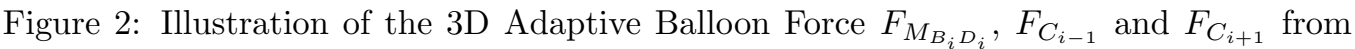
slices $i, i-1$ and $i+1$, respectively, where $i$ is the position on axis $z$.

first component defined in Eq. 9 uses the center point of its neighbors $M_{B_{i} D_{i}}$, as shown in red in Figure [2. This is achieved by averaging its neighboring points $B_{i}$ and $D_{i}$. Analyzing this point by the Jordan Curve Theorem (Berg et al, (1975), this point is taken as the internal point of the slice $i$, resulting in force $F_{M_{B_{i} D_{i}}}$ presented in yellow in Figure [1.

The second and the third components of force $F_{a d a p_{3 D}}$ are obtained from the nearest points of the neighboring slices by Eqs. ㅁ. and ㅁ. Eq. س defines the component from slice $i-1$, using the point closest to point $C_{i}$ defined in Figure $\nabla$ as being point $C_{i-1}$. This point is analyzed based on the Jordan Curve Theorem, Berg et al. (1975), changing the sign of Eq. 미 to positive, and pushing point $C_{i}$, as shown for force $F_{C_{i-1}}$ presented in green, so that it is inside the curve of slice i. Similarly, Eq. [ 1 uses point $C_{i+1}$ in the calculations so that this is the nearest to $C_{i}$ in slice $i+1$. In Figure 2, this point is internal to the curve of slice $i$, changing the sign of Eq. ㅁ] to positive, which causes force $F_{C_{i+1}}$, displayed in blue in Figure $\mathbf{2}$, to push point $C_{i}$.

The movement of each point in the proposed 3D model is therefore influenced by the curves in the neighboring slices whilst in the $2 \mathrm{D}$ model, only slice $i$ is analyzed by expanding the curve in this slice (Figure $\nabla$ ). Conse- 
quently, the movement of each point of the 3D model towards the objects of interest resultant from the proposed energy is optimized as more information is taken into account.

\subsection{D Adaptive Crisp External Energy}

The 3D Adaptive Crisp External Energy (3D ACEE) detects the origin of the edges of the lungs based on the analysis of pulmonary densities in the neighborhood of a voxel along with a Multi Layer Perceptron (MLP) artificial neural network to determining the origin of the edges found in the 3D traditional external energy, which in this work is based on the Sobel gradient in 3D (Al-Dossary and Al-Garni, 2013).

Starting from the Analysis of Pulmonary Densities (APD) (Rebouças Filho et al., 201]) method performed in a 3D neighborhood of a voxel, the percentages of 6 classes vi, in which $i$ varies from 0 to 5, are: air hyperinflated (1000 to $950 \mathrm{HU}$ ), normally air inflated (950 to $500 \mathrm{HU}$ ), low air inflated (500 to $100 \mathrm{HU}$ ), non-air inflated (100 to $100 \mathrm{HU}$ ), bone (600 to $2000 \mathrm{HU}$ ) and areas not classified, which are the densities that do not fit in the previous ranges. From the definition of these classes, a CT lung is considered as a set of overlapping images, i.e. slices. This analysis $T$ has dimension $l \times c \times a$, where $l \times c$ is the dimension of the slices and $a$ the number of slices of the exam under study.

Considering that the voxel under analysis has coordinates $(x, y, z)$, the function that determines the number of voxels with densities present in each class $v_{i}$ is defined as:

$$
f\left(x, y, z, v_{i}\right)=\sum_{l=-n}^{n} \sum_{m=-n}^{n} \sum_{o=-n}^{n} R(x-l, y-m, z-o),
$$

where $n$ is the size of the analyzed neighborhood and $R(x, y, z)$ is given by:

$$
R(x, y, z)=\left\{\begin{array}{l}
1, \lim _{\text {inf }}\left(v_{i}\right)<T(x, y, z)<\lim _{\text {sup }}\left(v_{i}\right), \\
0, \text { otherwise, }
\end{array}\right.
$$

where $\lim _{\text {inf }}\left(v_{i}\right)$ and $\lim _{\text {sup }}\left(v_{i}\right)$ are the lower and upper limits of the density range, in $\mathrm{HU}$, for the class $v_{i}$.

Using Eq. ए2, it becomes possible to calculate the percentage $P_{i_{3 D}}$ of each class $i$ as:

$$
P_{i_{3 D}}(x, y, z)=\frac{f\left(x, y, z, v_{i}\right)}{\sum_{j=0}^{4} f\left(x, y, z, v_{i}\right)} .
$$


After exhaustive testing, it was concluded that by increasing $n$, the image detection quality is increased, because the neighborhood size is proportional to $n$. However, a value of $n$ above 7 increases the processing time considerably, without any significant improvements in the results. Therefore, the value 7 was used for $n$ in the experiments.

The new 3D external energy uses an MLP artificial neural network in order to determine the origin of each edge found in the CT scans of the thorax. This neural network has, as inputs, the percentage of each class vi found by the ADP method (Rebouças Filho et al., 2010), and a topology of $6 / 4 / 1$ (Rebouças Filho et al., 20133). Its output indicates if an edge found in the thorax CT image belongs to the lung wall or not. Thus, a database is built from the voxel percentages extracted from examinations of COPD, cystic fibrosis and healthy patients.

A dataset was built manually, searching for the greatest possible representation of lung structures. Hence, $10 \mathrm{CT}$ lung exams used as part of diagnostic investigations with approximately 5000 slices were analyzed. The percentage $P_{i_{3 D}}$ was extracted for 500 voxels in each slice. Each set of inputs for these percentages was labeled, indicating which of the edges found in the 3D traditional external energy belonged to lung walls and which did not. Emphasizing that the 3D traditional external energy was calculated using the Sobel 3D operator, which calculates an average of the gradients found throughout the neighborhood being analyzed. The dataset built was validated by a cross-validation method (Haykin, 1999).

The following function is the output of the MLP network in execution, before its training phase:

$$
f_{m l p_{3 D}}(v)= \begin{cases}1, & \text { edge similar to lung wall, } \\ 0, & \text { otherwise }\end{cases}
$$

where $v$ consists of the 6 percentages $P_{i_{3 D}}$, where $i$ varies from 0 to 5 .

Using $f_{m l p_{3 D}}$ in order to determine the origin of the edges found in the CT lung images, the external energy is given by:

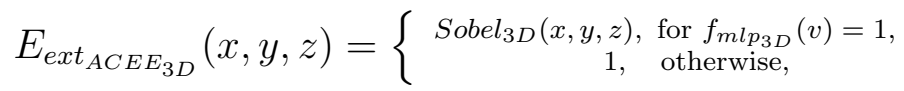

and $v$ is the percentage vector of the ADP 3D method obtained from Eq. ए2 using coordinates $(x, y, z)$ from the analyzed voxel.

Using Eq. [6], the MLP network determines the walls that are lung edges or not returning the value 1 using the function $f_{m l p_{3 D}}$. When this function 
returns the value 0 , it indicates that it is not a lung edge, and then the associated region receives the maximum crisp adaptive energy. Small objects presented only in a few number of slices now have less importance in the $3 \mathrm{D}$ model than in the related $2 \mathrm{D}$ model, as the energy is calculated with information from multiple slices.

\subsection{D Adaptive Crisp ACM Automatic Initialization}

The 3D model automatically starts inside the lungs. The method determines the initialization voxels in the right and left lungs, called 3D Righthand initial voxels $\left(R I P_{3 D}\right)$ and $3 \mathrm{D}$ Left-hand initial voxels $\left(L I P_{3 D}\right)$, respectively. Each one of these voxels has coordinates $\left(x_{i n i}, y_{i n i}, z_{i n i}\right)$. To carry this out, all the slices on the input CT scan are analyzed by the 2D initialization method in order to determine the exact initialization voxels (Rebouças Filho et al, 2013). The values of the $z$ coordinates of all slices that find a successful 2D automatic initialization are stored, then the average coordinate is adopted as $z_{i n i}$ and coordinates $\left(x_{i n i}, y_{i n i}\right)$ obtained by the $2 \mathrm{D}$ method of this slice are used as the initialization coordinates of voxels $R I P_{3 D}$ and $L I P_{3 D}$. As such, the method tends to start in the center of each lung.

Figure B] shows two curves presented in individual slices, where the distance between each voxel, in red, from the center of the 3D model is given by $R$; the blue line shows the centroid used in all slices and $r$ is the radius of the slice separated by a distance $d_{z}$ of the plane $z_{i n i}$ in the center of the slice $I$ considering only the axis $z$.

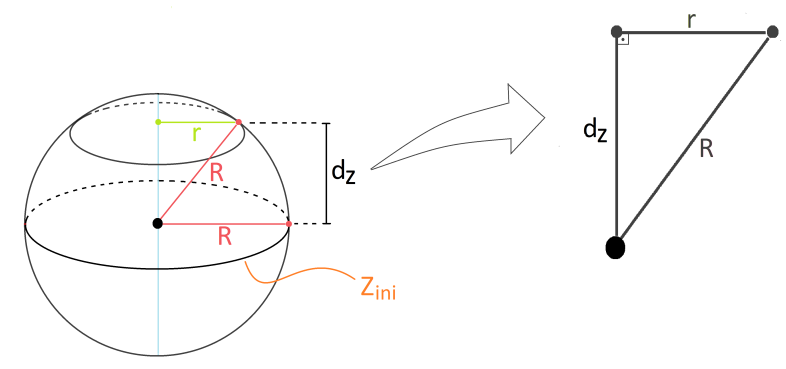

Figure 3: Definition of the initialization parameters for the 3D model.

Figure 3 depicts that each slice has a different distance from the centroid for each voxel. So, given slice $z_{i n i}$ that belongs to the center of the 3D model, it follows that the distance from the centroid for each voxel is the actual value. 
When the curve is on another slice, value $r$ must be calculated that is the radius of this further slice, and this value decreases as $d_{z}$ increases.

The algorithms used in the 3D display system used in this study were developed in $C$ and $C_{++}$languages and run on the OpenGL library (Astle and Hawkins, 20019; Sellers et aL, 2015). The GLU library is used to represent objects in 3D. GLUT library is used to create windows and receive user commands, and is multiplatform (Astle and Hawkins, 20009; Sellers et al, 2015). Figure $\mathbb{4}$ shows an automatic initialization example of the 3D model, where voxels $R I P_{3 D}$ and $L I P_{3 D}$ were found in a CT scan with 900 slices. This initialization took 15 slices, using 30 voxels in each slice and a distance of 30 voxels between each voxel and the center of each lung, in which the starting voxels were $R I P_{3 D}$ and $L I P_{3 D}$ of the right and left lung models, respectively. These lung models are shown in "lung" color in Figure 4(a), and Figure 4(b) displays one of the final lung models built.

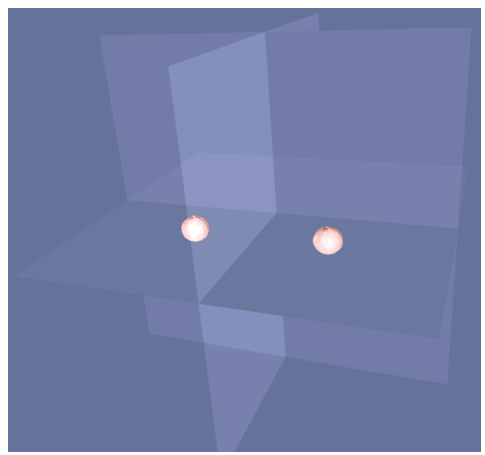

(a)

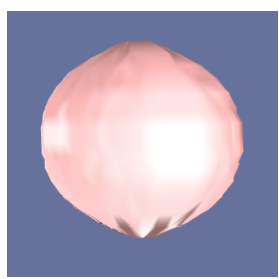

(b)

Figure 4: Automatic initialization of the 3D segmentation model in each of the lungs a) and one of the final models built b).

\subsection{Movement, Adding and Removing points}

In this section, the dynamics of displacement, adding and removing ACM points in the proposed 3D segmentation method are described. The displacement of points is analyzed in a unique neighborhood, and the point coordinates that generate a lower energy $E_{3 D}$ set are its new coordinates. Hence, the point coordinates are updated in order to minimize the total model energy given by Equation $\square$.

Figure 6 illustrates a $3 \times 3$ neighborhood of a point $c(s)$ belonging to a slice $i$ considering the axis $z$ of the 3D model. Slices $i-1$ and $i+1$ are only 
used for the calculation of the 3D energy that contributes to the total energy of the ACM Crisp 3D Adaptive $\left(E_{C A_{3 D}}\right)$. Thus, the displacement of a point belonging to a given slice will only occur in $z_{i}$ of this slice. Therefore, not only the points between distant neighbors in the same slice should be added, but also the points in neighboring slices. This is necessary so that the $3 \mathrm{D}$ model can detect an object that is present in several slices, and not all slices have a curve initially.
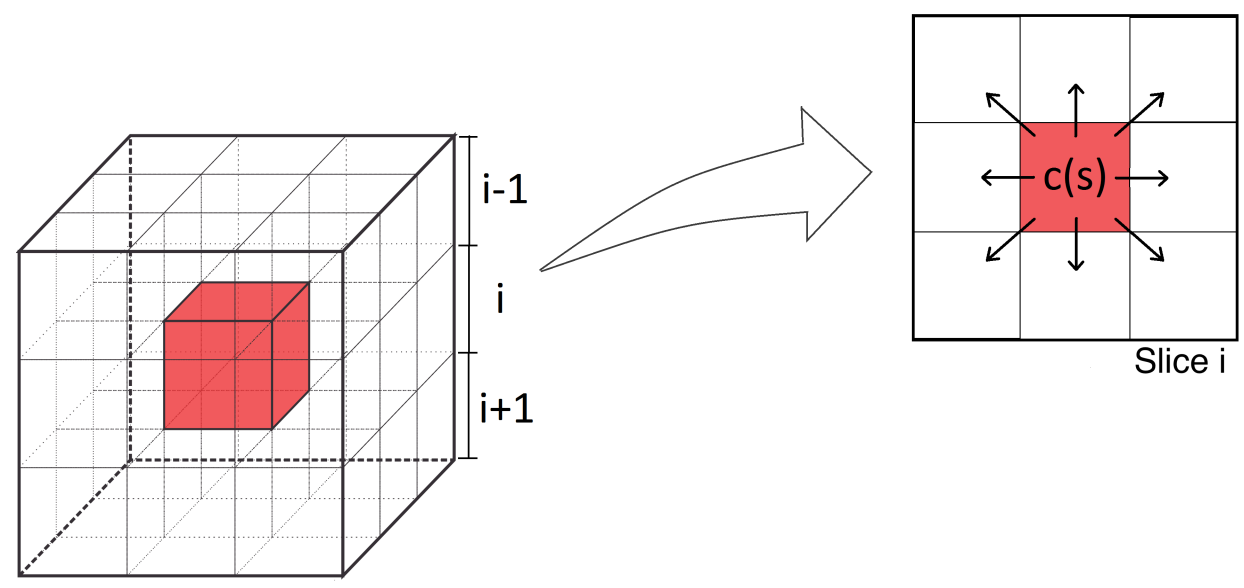

Figure 5: Illustration of a $3 \times 3$ neighborhood for the analysis of the energy and the movement of a point $c(s)$ belonging to slice $i$, wherein slices $i 1$ and $i+1$ are just used to define the shifting, addition and removal of points in a $3 \mathrm{D}$ model.

The addition of points occurs through two distinct methods. The first method adds points between neighborhoods of a slice that have a distance greater than the maximum allowed. The second method adds points between neighboring slices. This method analyzes the curves present in two slices, the first and last slices of the 3D model. This analysis is based on the perimeter of these curves, wherein a maximum circumference $\left(P_{\max }\right)$ is initially configured, and the method adds a new curve where one of these aforementioned curves exceeds the value of the predefined $P_{\max }$.

Considering that the first slice is slice $f$ and the last one is slice $l$, then when the first slice has a perimeter larger than $P_{\max }$, a new curve is added to slice $f-1$. However, when the last slice of the model has a perimeter larger than $P_{\max }$, a new curve is added to slice $l+1$. This new curve is added using the centroid of the curve in the analysis as a reference. When adding a curve 
in slice $f-1$ it is used the centroid of $f$ and when adding a curve to slice $l+1$ the centroid of the curve from slice $l$ is used.

Figure [ illustrates the application of this method using 30 as the maximum perimeter $P_{\max }$ and the initialization parameters assuming the value 10 as the distance from each point to the centroid and 30 as the number of vertices. In Figure 6(a), the upper and lower slices have greater perimeters than $P_{\max }$; Figure Figure 6(b) shows the visualization of this model with an internal view of the top slice. The results of applying the points addition method in the upper and lower slices are shown in Figure 6(c). Figure 6(d) shows the external and internal views of the model, respectively, after the addition of the points.

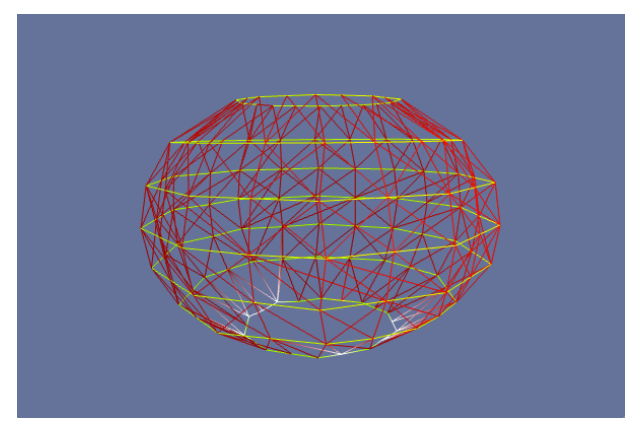

(a)

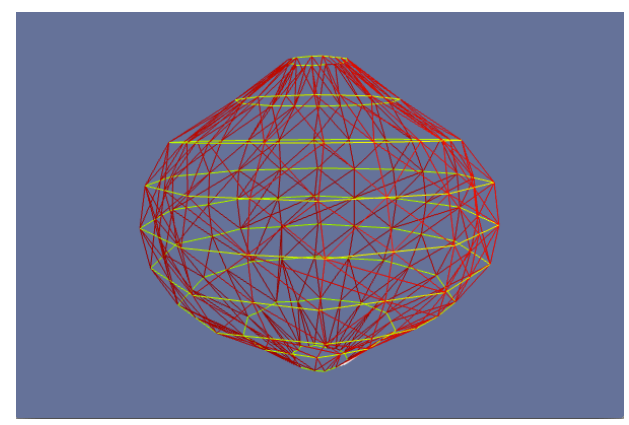

(c)

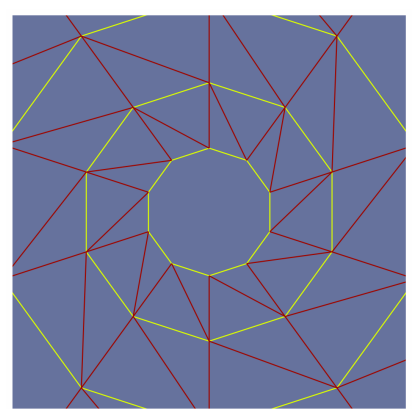

(b)

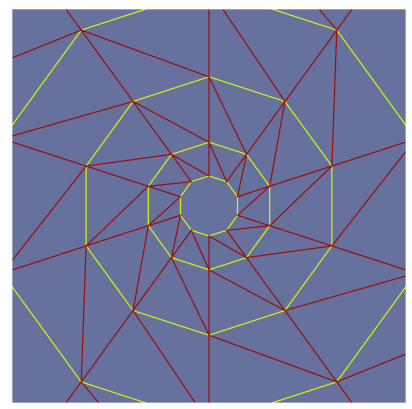

(d)

Figure 6: Adding slices to a 3D model: a) 3D model with areas larger than the ones defined in the first and last slices; b) top view of the model in a); c) 3D model after adding the new slices; and d) top view of the model in c).

Another important step in the proposed method is the removal of points from the 3D model. Analogously to the method of addition of points, this 
step is also based on two methods. The first method uses information of the neighbors of a point in the same slice as illustrated in Figure 7 . The angle formed between the analyzed point and its neighbors in this slice is calculated as $\alpha=\arccos \left(\frac{a^{2}+b^{2}-c^{2}}{2 b c}\right)$, and if this angle is less than a predefined angle, this point is removed from the model and model is reordered. In summary this method removes the model points that are misaligned with their neighbors.

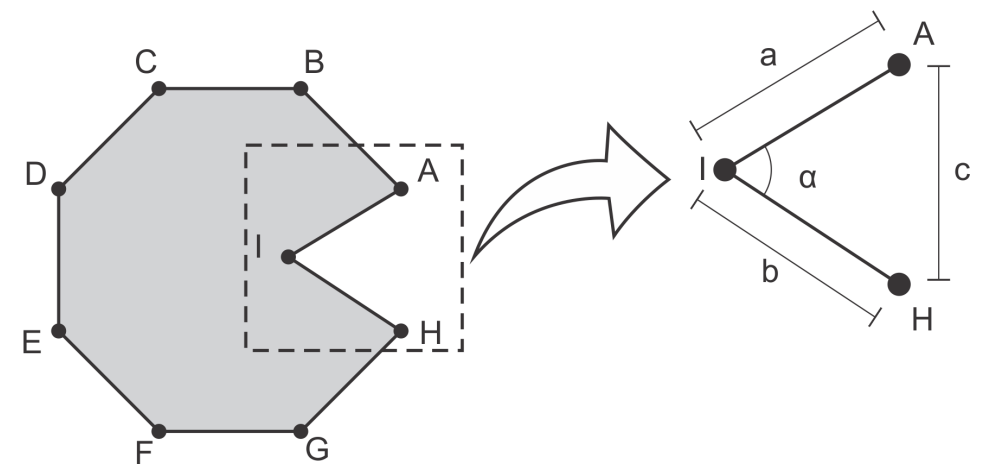

Figure 7: Calculation of the angle between a point and its neighbors on the same slice.

The second method follows the same principle to remove points from its neighbors, but expanding the principle to 3D. This is possible using the closest points in neighboring slices. Thus, considering a point belonging to slice $z_{i}$, the nearest point of slice $z_{i-1}$ and the closest point of slice $z_{i+1}$ used, as illustrated in Figure $\nabla$ with points $C_{i}, C_{i-1}$ and $C_{i+1}$ belonging to slices $i$, $i-1$ and $i+1$, respectively.

The analysis for the removal of points is based on the angle formed between the neighbor points that is compared with a predefined minimum angle $\theta_{\text {min }}$. An analyzed point is removed when the angle between the point and the closest ones in the neighboring slices is less than $\theta_{\text {min }}$. Given the model shown in Figure $\mathbb{8}$, one can see the point $C_{i}$ forming an angle $\theta$ with $C_{i-1}$ and $C_{i+1}$, where these are the closest points in slices $i-1$ and $i+1$, respectively.

Angle $\theta_{1}$ shown in Figure 8(a) is greater than angle $\theta_{2}$ in Figure 8(b). This is because $C_{i}$ is less aligned with $C_{i-1}$ and $C_{i+1}$ in the formation of $\theta_{2}$, which does not occur in the formation of $\theta_{1}$. The angle $\theta$ formed between a point and its closest points in the neighboring slices is given by $\theta=\arccos \left(\frac{a^{2}+b^{2}-c^{2}}{2 b c}\right)$, where $a, b$ and $c$ are the parameters identified in Figure $\mathbf{8}$.

Thus, the removal methods tend to exclude the misaligned points from 

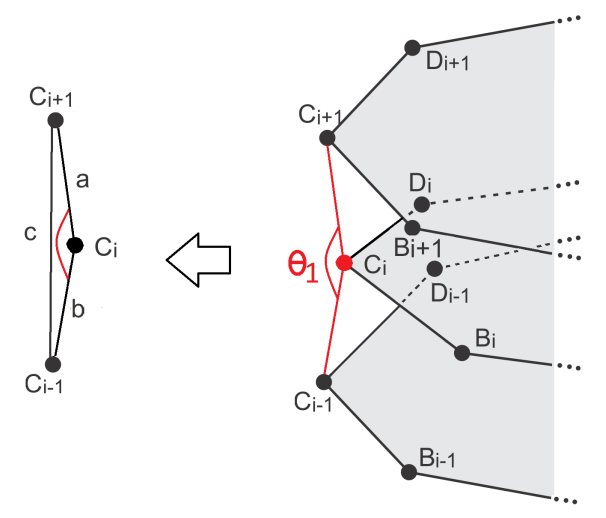

(a)
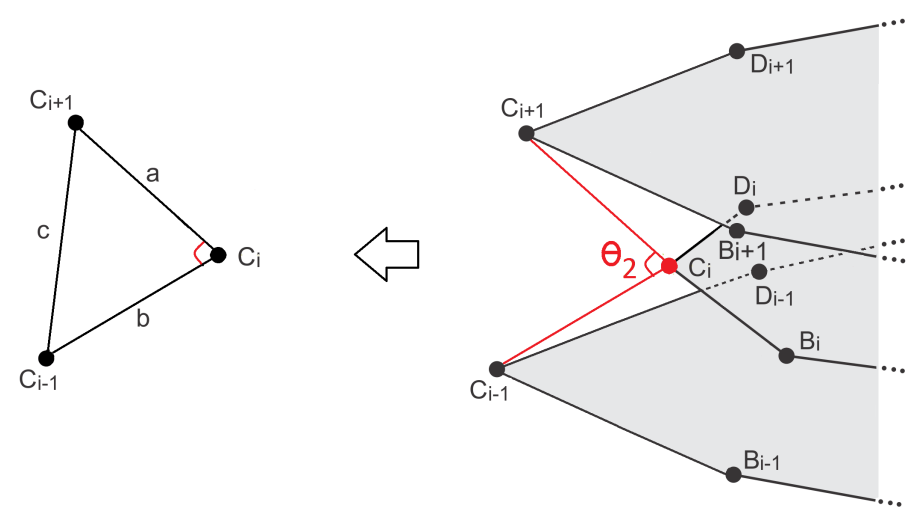

(b)

Figure 8: Illustration of the parameters used for calculating the angles formed between a point of slice $i$ with its nearest neighbor in slices $i-1$ and $i+1$ : a) and b) show the definition of angles $\theta_{1}$ and $\theta_{2}$, respectively.

the other slices. The points removed are points that are misalignment relative to their neighbors in the same slice or relative to the points in curves present in the neighboring slices. This makes the model smoother and avoids gross errors in the $3 \mathrm{D}$ segmentation.

\subsection{Automatic Segmentation of Lungs in Thorax CT Scans}

The automatic segmentation of the lungs in a CT scan of the thorax uses the methods previously described for the automatic initialization of the 3D model, addition and removal of points and the 3D Adaptive Crisp ACM. The 


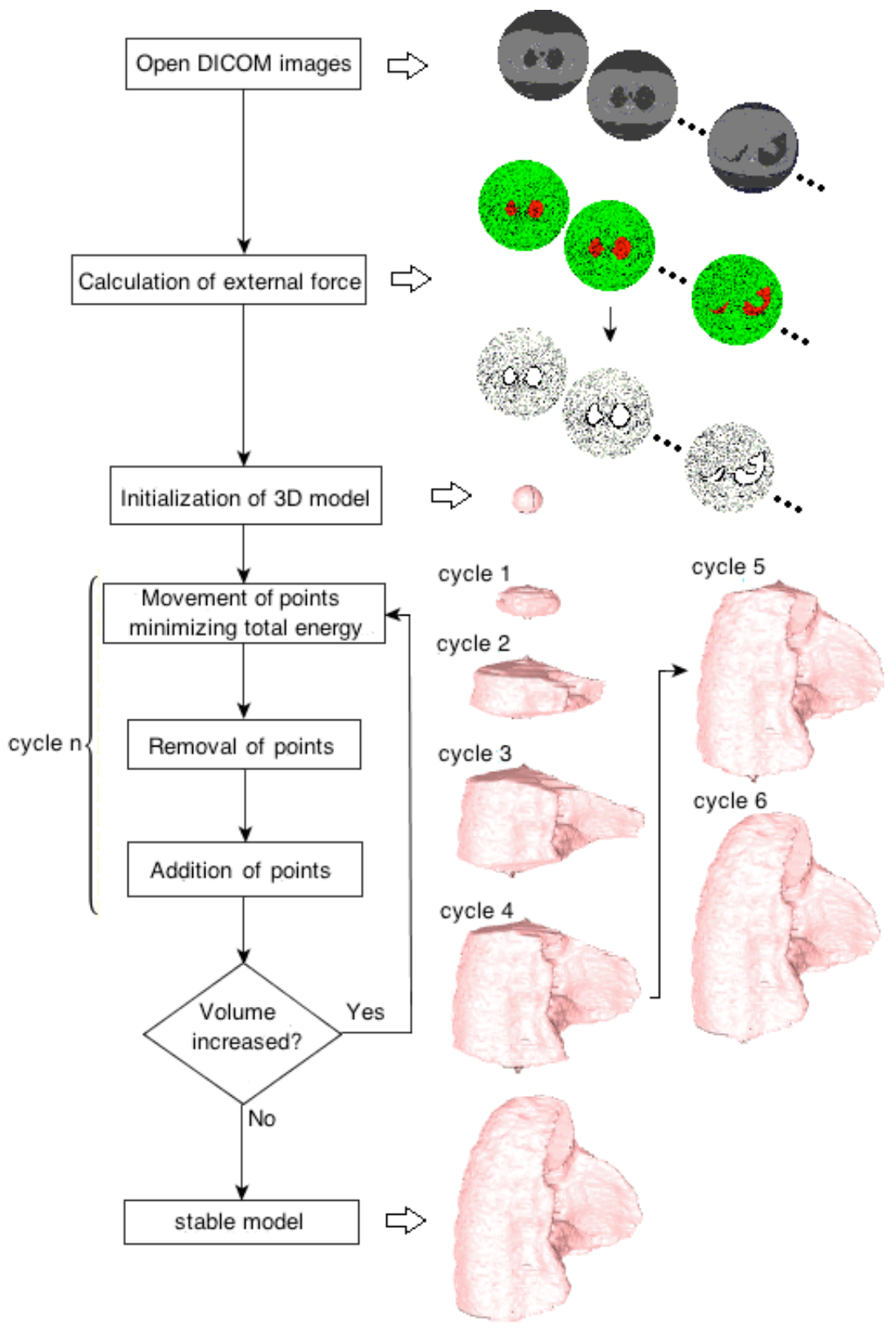

Figure 9:Flowchart for implementing the 3D Adaptive Crisp ACM.

methods are executed according to the flowchart shown in Figure Q1, which also includes examples related to each step involved. 
The first step in segmenting the lungs automatically in a CT examination is to open all the DICOM images. To carry this out, the free library DCMTK is used to read the image and its parameters and to identify and order the CT scan slices. Then, the whole external energy is calculated using the 3D Adaptive Crisp method for detecting the origin of the edges obtained by the 3D traditional external energy. The edges detected inside the lungs are excluded from the external energy. The centroid of the 3D model with coordinates $x_{i n i}, y_{i n i}$ and $z_{i n i}$ is determined by the slice of the average coordinate $z_{i}$, considering all slices $i$, where the lung was found using the 2D method suggested in Rebouças Filho et al. (2013). The 3D model under goes successive iterations of the 3D Adaptive Crisp ACM method in order to decrease the energy of the model by moving its points. In each of the iterations, the methods of 3D points removal and addition are applied.

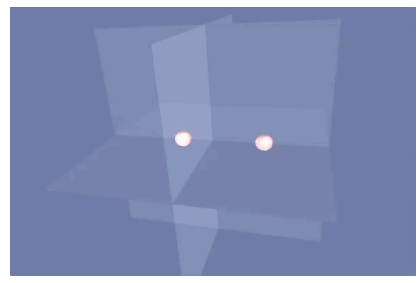

(a)

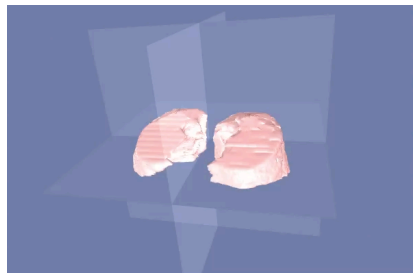

(d)

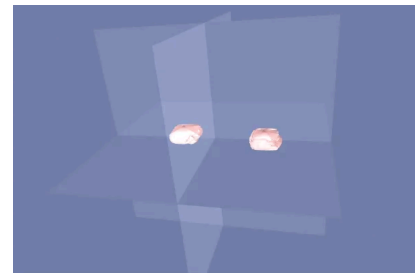

(b)

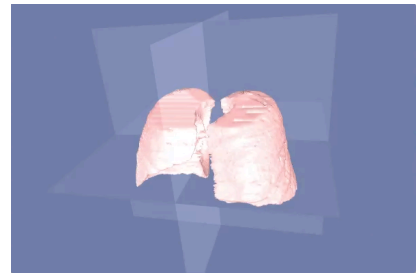

(e)

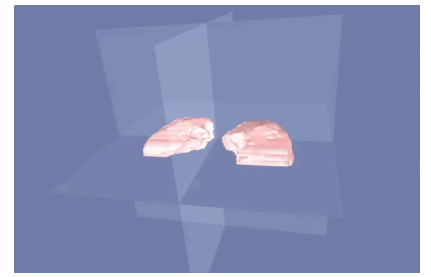

(c)

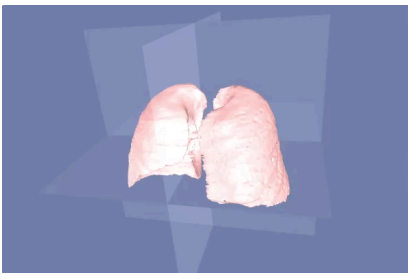

(f)

Figure 10: Lung segmentation in CT scans by 3D Adaptive Crisp ACM: a) automatic initialization of the 3D model; b) to e), evolution of the 3D model, and f) final result.

The model is stable when the volume does not increase after two consecutive iterations. When this happens, the segmentation of the lung is

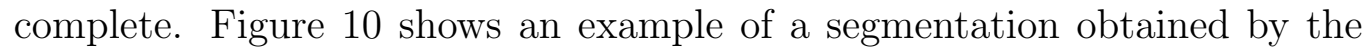
proposed method, from the initialization to the stabilization of the 3D model. 


\subsection{Statistical measures}

In order to analyze the segmentation performance, three well-known measures were employed: recall, precision and F-measure, whose definitions are briefly described here:

Recall (aka Sensitivity) is the ratio between the number of correctly segmented voxels of a given class and the total number of voxels in the CT scan of the thorax under analysis, including those that were incorrectly segmented:

$$
\text { Recall }=\frac{\text { true positives }}{\text { true positives }+ \text { false negatives }},
$$

where true positives and false negatives stand for the number of voxels of a given class correctly and incorrectly segmented, respectively.

Precision (aka Positive predictive value) means the ratio between the number of correctly segmented voxels of a specific class and the total number

of voxels in the CT scan of the thorax under analysis as belonging to that class:

$$
\text { precision }=\frac{\text { true positives }}{\text { true positives }+ \text { false positives }},
$$

where true positives and false positives denote the number of voxels correctly and incorrectly segmented as belonging to the considered class, respectively.

The F-measure $\left(F_{m}\right)$ for a given class is calculated as the harmonic mean of the Recall and precision values for that specific class, resulting in a more global parameter for evaluating the performance of a classifier on each class. More formally:

$$
F_{m}=2\left(\frac{\text { Recall } \times \text { precision }}{\text { Recall }+ \text { precision }}\right) .
$$

\section{Experimental Results}

In this section, we present the results in terms of computational cost and performance of each lung segmentation method under comparison. The tests were performed on a notebook with an Intel Core i5 $1.4 \mathrm{GHz}, 4 \mathrm{~GB}$ of RAM, and running MAC OS X 10.10.5.

In the evaluation, the computational cost (processing time), positive predictive value (precision), sensitivity (recall) and F-measure were used to calculate the similarity between the shapes under comparison. 
Table 1: Description of the CT chest exams used to analyze the 3D algorithms.

\begin{tabular}{c|c|l}
\hline $\begin{array}{c}\text { CT scan } \\
\text { number }\end{array}$ & $\begin{array}{c}\text { Number of } \\
\text { images }\end{array}$ & Pathology \\
\hline \hline 1 & 456 & Normal \\
2 & 282 & Normal \\
3 & 269 & Normal \\
4 & 301 & Normal \\
5 & 344 & COPD \\
6 & 382 & Normal \\
7 & 241 & Fibrosis \\
8 & 232 & Normal \\
9 & 279 & Fibrosis \\
10 & 299 & Normal \\
11 & 299 & COPD \\
12 & 229 & Bronchiectasis \\
13 & 228 & Bronchiectasis \\
14 & 220 & Bronchiectasis \\
15 & 268 & Nodular calcification \\
16 & 260 & Normal \\
17 & 248 & COPD \\
18 & 224 & Bronchiectasis and Fibrosis \\
19 & 228 & Nodular calcification and COPD \\
20 & 276 & calcification \\
21 & 224 & Fibrosis \\
22 & 224 & Parenchymal bands \\
23 & 228 & Bronchiectasis and calcifications \\
24 & 240 & Bronchiectasis and calcifications \\
25 & 256 & Fibrosis and calcifications \\
26 & 228 & Bronchiectasis \\
27 & 180 & Bronchiectasis and Fibrosis \\
28 & 256 & Normal \\
29 & 224 & Bronchiectasis and Fibrosis \\
30 & 256 & Normal \\
31 & 300 & Parenchymal bands \\
32 & 256 & Calcification and COPD \\
33 & 232 & Bronchiectasis and Parenchymal bands \\
34 & 228 & Calcification and COPD \\
35 & 248 & Normal \\
36 & 244 & Normal \\
37 & 224 & Bronchiectasis and calcifications \\
38 & 252 & Bronchiectasis and calcifications \\
39 & 268 & Bronchiectasis \\
40 & 264 & Atelectasis and COPD \\
\hline & & \\
\hline
\end{tabular}

\subsection{Images Acquisition}

The CT systems used to acquire the experimental 40 chest CT scans of healthy volunteers and patients with various types of pathology were of high resolution. The acquired images have a resolution of $512 \times 512$ with 16 bits.

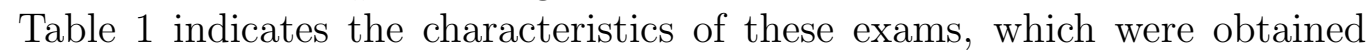
in partnership with the Walter Cantidio University Hospital, in Brazil, and used in an earlier study (Rebouças Filho et al., 2017, 2013).

\subsection{Definition of the optimal parameters}

In this section, the definition of the optimal parameters to be used with the proposed method for segmenting the lungs in the experimental CT scans of the chest is described.

The energies involved in a parametric active contour model, either in $2 \mathrm{D}$ or $3 \mathrm{D}$, need to be parameterized and each energy component has a different 
importance in the calculation of the total energy for each pixel or voxel. As such, the parameters $\alpha, \beta$ and $\tau$ define the weights of the 3D Adaptive Balloon Force, 3D Continuity Force and 3D Adaptive Crisp External energy, respectively, in the calculation of the total energy.

The optimal parameters were defined by cross-validation varying each parameter and considering the sum of all as equal to 1 (one). The graphs of Figure $\square$ depict the results of the F-measures and processing times. Three columns are shown for three different weights for 3D Continuity Force, the gray column is $\beta=0.5$, black column $\beta=1.0$ and white column $\beta=1.5$. The $\mathrm{x}$-axis shows the values of parameter $\alpha$ and the $\tau$ value is the complement of the sum between $\alpha$ and $\beta$ to $1(\tau=1-\alpha-\beta)$. These results were obtained using $6 \mathrm{CT}$ scans considering different clinical cases: normal (CT scans 1 and 4), calcification (CT scans 15 and 20), bronchiectasis (CT scan 26), and parenchymal bands (CT scan 31).

Analyzing Figure 11(a), one can verify that if alpha is increased, the F-measure value will increase until it stabilizes; yet, it starts to drop from $\alpha=0.5$. However, analyzing Figure 11(b), one can see that the higher the alpha value is, the faster the lung segmentation in CT scans tends to be. Therefore, the optimal configuration was obtained using $\beta=0.05$ (grey column) with $\alpha=0.60$, and $\tau=0.35$, as indicated by the red line shown in Figure $\mathbb{~}$ ]. This option will lead to the shortest possible processing time and highest efficiency.

\subsection{Numerical contribution of the proposed 3D method compared to the $2 D$ method}

To evaluate numerically the contribution of the proposed $3 \mathrm{D}$ method compared to the 2D method proposed in Rebouças Filho et al. (2013), we used the optimal configuration obtained for both methods on the same 6 CT scans (Figure $\square$ ). The 2D method obtained an F-measure of $96.33 \% \pm 0.42$ and a processing time of $12.52 \pm 2.10$ minutes. The proposed 3D Adaptive Crisp Active Contour obtained an F-measure of $99.14 \% \pm 0.18$ and a processing time of $3.20 \pm 0.38$ minutes. These results demonstrate that the novel 3D energy accelerates the convergence of the $3 \mathrm{D}$ model, thus reducing the processing time, with the combination of each new 3D energy making the proposed 3D method 3.91 times faster than the 2D method compared under the same experimental settings and conditions.

The use of the Sobel 3D operator resulted in the F-measure average value obtained by the proposed 3D model being 3.5\% higher than the one obtained 


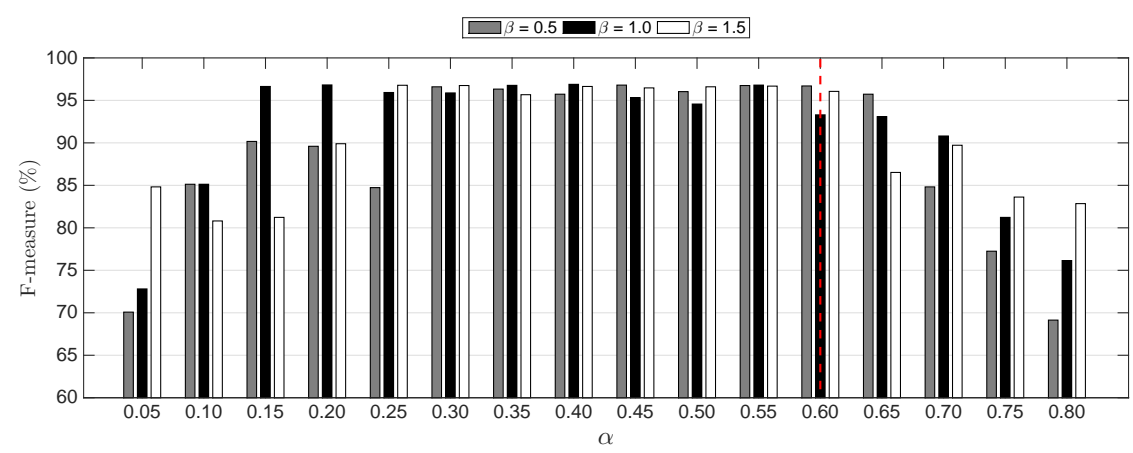

(a)

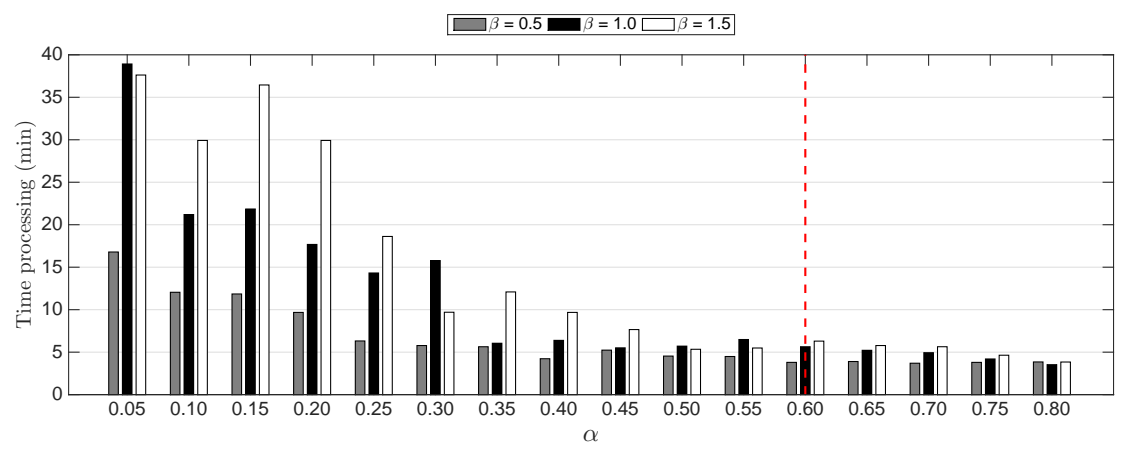

(b)

Figure 11: Results of the F-measure and processing time obtained by cross-validation varying $\alpha, \beta$ and $\tau$ in order to define the weights of the 3D Adaptive Balloon Force, 3D Continuity Force and 3D Adaptive Crisp External energy, respectively. The grey bar column is $\beta=0.5$, black column $\beta=1.0$ and white column $\beta=1.5$. The optimal configuration is indicated by a red line.

by the 2D method. Also the proposed 3D method is more stable, which can be confirmed by analyzing the standard deviations of the F-measure.

\subsection{Results and Discussion}

In the experimental assessment, the 3D ACACM was compared against three common approaches used in this field: the automatic 3D Region Growing (3D RG) algorithm, the level-set algorithm based on the coherent propagation method (LSCPM) and the semi-automatic segmentation performed by an expert using the 3D OsiriX toolbox (EUOT). 
The 3D Adaptive Crisp ACM method was configured with the parameters (as described in Section [3.2) $\alpha=0.60, \beta=0.05$ and $\tau=0.35$ in the calculation of the total energy. After the initialization, the centroids were determined. To build the initial 3D model, 30 voxels per slice with a radius value of 50 voxels for the distance to the centroid were used. The maximum distance $d$ among voxels considered in the addition of new voxels was equal to 5 voxels and the minimum angle between a voxel and its neighbors considered in the removal of voxels was defined as 45 degrees.

The 3D RG algorithm used the same initialization as 3D Adaptive Crisp ACM, with the entire internal region of the ACM initialization polygon used as seed. The neighboring regions addition method uses lung anatomy information, only adding voxels that are on intensity edges within the lung, which are: normally aerated, slightly aerated or hyper-inflated. This addition occurs by successive iterations, ending when no more voxels can be added. Two updates are made in this method. First of all, the trachea and the hilum are targeted separately by removing the voxels of this region from the result of the segmentation of the lungs. Finally, if the regions of both lungs are tending to merge, the frontier between the two lungs is updated to avoid segmenting regions of one lung as of the other lung. This frontier is moved to the location of the smallest diameter between the regions. Assuming that the voxels where the two lungs merge looks like an hourglass, the frontier is moved to the middle of the hourglass.

There are several types of commercial medical software with plugins and toolboxes that can be used to compare the proposed method. We used one that is mostly used in hospitals and also in recent researches. Hence, the levelset algorithm based on the coherent propagation method (LSCPM), proposed by Wang et al. (2011, 2014), was used in this work for lung segmentation via the MIA plugin for OsiriX (http://www.mia-solution.com).

Another segmentation approach possible is the semi-automatic segmentation by an expert using the 3D OsiriX toolbox (EUOT). In this approach, the expert visualizes the 3D objects presented in the input exam, and removes undesired objects (Michael P (hae, 2015). The use of EUOT is in fact widely adopted by many doctors; however, this tool is based on simple segmentation techniques such as thresholding and region growing. Thus, when the lung under analysis has some disease, manual corrections should be made in each slice of the CT dataset; however, this tool does not allow this straightforward procedure. Figure 12 shows examples of the segmentations obtained by the methods under comparison. 


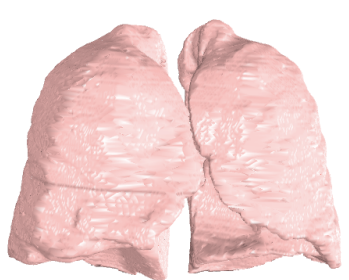

(a)

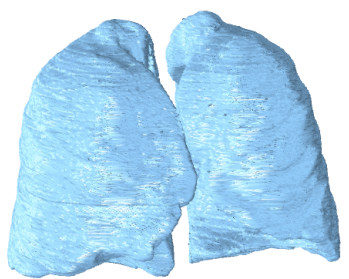

(d)

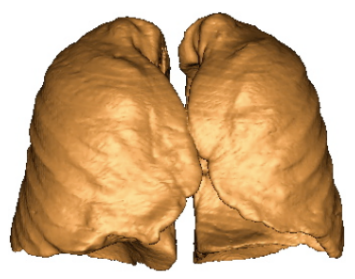

(g)

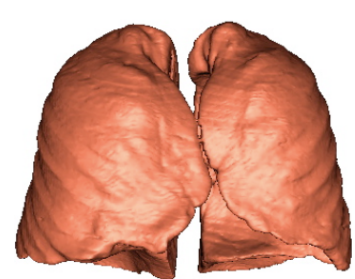

(j)

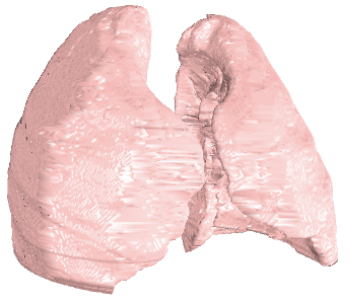

(b)

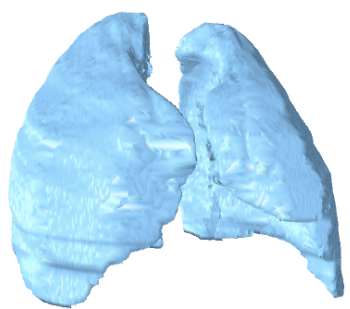

(e)

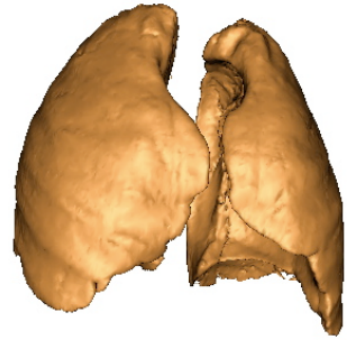

(h)

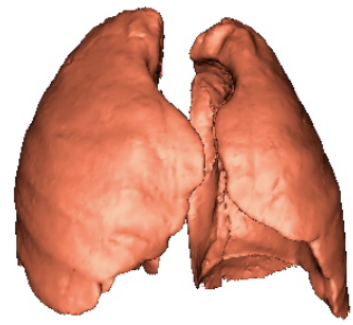

(k)

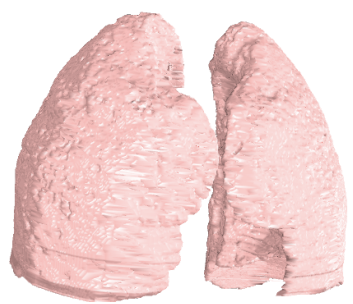

(c)

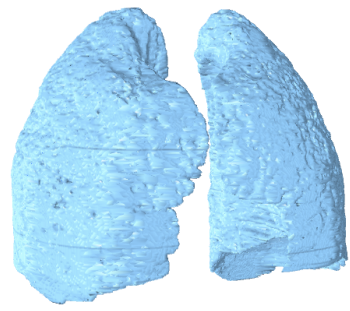

(f)

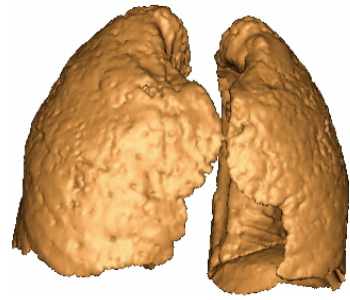

(i)

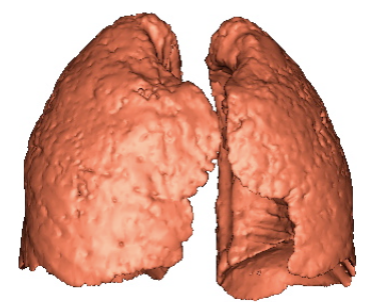

(1)

Figure 12: Lung segmentation in CT scans by the methods under comparison: a), b) and c) 3D Adaptive Crisp ACM; d), e) and f) 3D Region Growing; g), h) and i) Levelset algorithm based on the coherent propagation method; j), k) and l) semi-automatic segmentation by an expert using the 3D OsiriX toolbox. 
The segmentations obtained by each method under comparison were evaluated using $40 \mathrm{CT}$ scans of the chest. Each CT scan was assessed along its length from the apex to the base of the lung, removing one in eight slices.

The segmentations used as ground truth were built semi-automatically using commercial software and manual corrections were subsequently carried out by a medical expert on the existing errors.

Table 7 shows the statistical results obtained for the $40 \mathrm{CT}$ scans in terms of healthy patients and patients with diseases. In the case of the diseased CT scans, there are two groups, the first group with decreased HU density in some regions of the lung such as those due to COPD and bronchiectasis, and the second group with increased HU densities such as those due to fibrosis, calcification and atelectasis.

Table 2: Statistical analysis of the F-measure (FM) values obtained for the lung segmentation methods on the experimental CT scans in terms of healthy lungs (HL), lungs with disease that increase or decrease the HY density (DID and DDD, respectively), and global results (GR).

\begin{tabular}{c|c|c|c|c|c} 
& Segm. & Right lung & Left lung & \multicolumn{2}{c}{ Both lungs } \\
& Method & FM $(\%)$ & FM $(\%)$ & FM $(\%)$ & Time $(m i n)$ \\
\hline \multirow{4}{*}{ HL } & 3D ACACM & $\mathbf{9 9 . 9 3} \pm \mathbf{0 . 1 9}$ & $\mathbf{9 8 . 8 2} \pm \mathbf{0 . 2 2}$ & $\mathbf{9 9 . 2 2} \pm \mathbf{0 . 1 4}$ & $\mathbf{3 . 5 4} \pm \mathbf{0 . 7 9}$ \\
& 3D RG & $98.19 \pm 0.74$ & $98.28 \pm 0.77$ & $98.57 \pm 0.54$ & $2.51 \pm 0.55$ \\
& EUOT & $98.03 \pm 1.16$ & $97.92 \pm 1.27$ & $98.53 \pm 0.99$ & $4.38 \pm 0.89$ \\
& LSCPM & $98.26 \pm 1.25$ & $98.08 \pm 1.36$ & $98.73 \pm 1.04$ & $1.18 \pm 0.26$ \\
\hline \multirow{5}{*}{ DDD } & 3D ACACM & $\mathbf{9 8 . 9 0} \pm \mathbf{0 . 1 1}$ & $\mathbf{9 8 . 6 9} \pm \mathbf{0 . 2 3}$ & $\mathbf{9 9 . 1 9} \pm \mathbf{0 . 0 8}$ & $\mathbf{3 . 0 4} \pm \mathbf{0 . 4 7}$ \\
& 3D RG & $98.40 \pm 0.55$ & $98.16 \pm 0.84$ & $98.70 \pm 0.43$ & $2.15 \pm 0.33$ \\
& EUOT & $98.02 \pm 1.01$ & $97.68 \pm 1.35$ & $98.65 \pm 0.84$ & $3.72 \pm 0.56$ \\
& LSCPM & $98.07 \pm 1.14$ & $97.65 \pm 1.50$ & $98.76 \pm 0.92$ & $1.01 \pm 0.15$ \\
\hline \multirow{5}{*}{ DID } & 3D ACACM & $\mathbf{9 8 . 9 4} \pm \mathbf{0 . 2 5}$ & $\mathbf{9 8 . 7 8} \pm \mathbf{0 . 2 8}$ & $\mathbf{9 9 . 2 2} \pm \mathbf{0 . 1 6}$ & $\mathbf{2 . 9 1} \pm \mathbf{0 . 3 2}$ \\
& 3D RG & $94.49 \pm 7.61$ & $93.49 \pm 10.67$ & $96.43 \pm 5.06$ & $2.06 \pm 0.22$ \\
& EUOT & $93.78 \pm 8.64$ & $92.68 \pm 11.35$ & $96.08 \pm 5.97$ & $3.69 \pm 0.38$ \\
& LSCPM & $92.92 \pm 10.21$ & $91.74 \pm 13.43$ & $95.56 \pm 7.46$ & $0.97 \pm 0.10$ \\
\hline \multirow{4}{*}{ GR } & 3D ACACM & $\mathbf{9 8 . 9 4} \pm \mathbf{0 . 2 1}$ & $\mathbf{9 8 . 7 7} \pm \mathbf{0 . 2 6}$ & $\mathbf{9 9 . 2 2} \pm \mathbf{0 . 1 4}$ & $\mathbf{3 . 1 1} \pm \mathbf{0 . 5 7}$ \\
& 3D RG & $96.50 \pm 5.60$ & $95.99 \pm 7.36$ & $97.59 \pm 3.67$ & $2.20 \pm 0.40$ \\
& EUOT & $96.01 \pm 6.37$ & $95.39 \pm 8.28$ & $97.39 \pm 4.35$ & $3.89 \pm 0.66$ \\
& LSCPM & $95.68 \pm 7.56$ & $94.98 \pm 9.81$ & $97.22 \pm 5.43$ & $1.03 \pm 0.19$ \\
\hline
\end{tabular}

This study adopted the F-measure as the quality metric because this measure takes into account only the lung region in the calculations. Many authors use the accuracy as an evaluation metric, but in this case, this metric does not lead to accurate results, since the lung is just a small part of the total imaged volume, and accuracy considers the whole volume under examination, 
which can lead to erroneous analysis of the results.

Regarding the results presented in Table $\boldsymbol{Z}$, there are four analyzes. The first one evaluates healthy patients, and here the average F-measure by the proposed method is better for both lungs with a value of $99.22 \% \pm 0.14$, followed by the Level-set algorithm based on the coherent propagation method with a value of $98.73 \% \pm 1.04$ for this metric, then the $3 \mathrm{D}$ RG with $98.57 \% \pm$ 0.54 and finally, the semi-automatic segmentation by an expert using 3D OsiriX toolbox with $98.53 \% \pm 0.99$.

The second analysis concerns patients with diseases. The presence of exams with diseases that decrease the HU density in some lung regions does not change significantly the results of the methods: as to the average Fmeasure, the 3D ACACM has a value of $99.19 \% \pm 0.08$, LSCPM of $98.76 \% \pm$ $0.92,3 \mathrm{D} R \mathrm{RG}$ of $98.70 \% \pm 0.43$, and EUOT of $98.65 \% \pm 0.84$. However, this behavior does not occur for diseases that increase the HU density in some regions of the lung. In these cases, the 3D ACACM results remain practically constant with a value of $99.22 \% \pm 0.16$ for the average F-measure; however, the other methods had lower performances: 3D RG obtained a value of $96.43 \% \pm 5.06$, LSCPM of $95.56 \% \pm 7.46$, and EUOT of $96.08 \% \pm 5.97$. Figure 1.3 presents boxplots of the most critical case associated to the existing methods, i.e. the segmentation of lung diseases that tend to increase the values of HU tissue density.

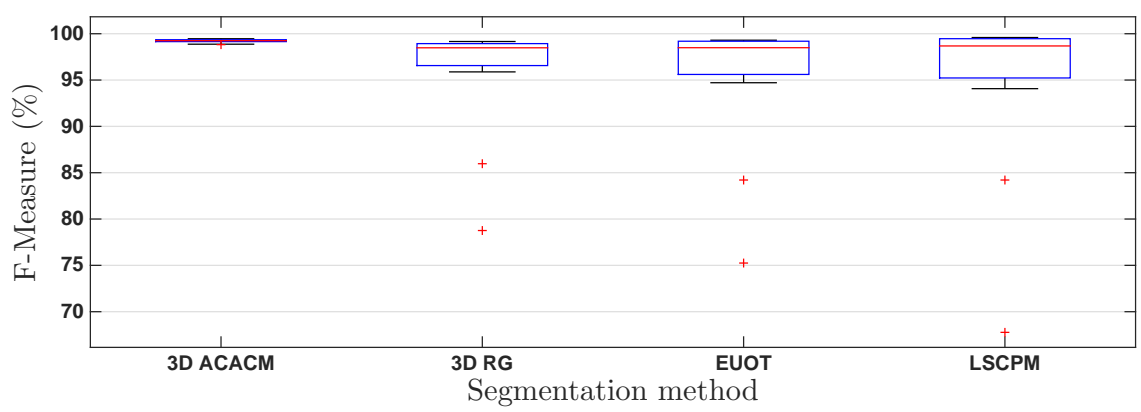

Figure 13: F-measure (FM) boxplots of the values obtained for the lung segmentation methods on the experimental CT scans in terms of disease that increase the HU density (DID).

The boxplots in Figure [3] show that in cases of DID, the existing methods have considerably variations of hit rate. This is because these diseases make 
the unhealthy lung tissue very dissimilar to healthy lung tissue. However, the proposed method remains robust with an almost constant hit rate, obtaining results similar for healthy volunteers and patients with DDD and DID.

Figure 14 shows the results considering healthy and unhealthy lungs and illustrates common errors that can occur in this field. The results are presented according to the following: the first row shows healthy lungs; the second row shows lungs with COPD; the third row presents lungs with Bronchiectasis and Parenchymal bands; the fourth row shows lungs with Bronchiectasis and Fibrosis; the fifth row shows lungs with Nodular calcification and COPD. The results are presented with true positives in green, false negatives in red, and false positives in orange and blue (blue is used in the cases where the border between the 2 lungs is unclear), and the original grayscale represents true negatives.

Regarding Figure 14, the results in the first row indicate that all methods obtained correct segmentations. The slices in the third, fourth and fifth rows, are associated to lung diseases that increase the HU density which confounds inside lung regions with outer lung regions. The methods compared against the proposed 3D ACACM method had lower performance in segmenting these slices. Instead, the proposed method had a stable performance in all of these cases due to its external energy and integration of artificial intelligence that enhances its performance even more. The internal energy makes segmentation of objects with different shapes possible, building the correct $3 \mathrm{D}$ segmented model due to its integration with the appropriate external force.

In the fifth row of Figure 14, there are small blue regions in the results obtained by the existing methods, meaning an uncertainty of where the border between the two lungs is; however, the proposed method performed very well also due to the adopted external energy. Furthermore, the proposed internal energy becomes more regular and stable in the 3D model and thus reduces gross segmentation errors. Note that this behavior of the 3D ACACM energy can also cause minor errors, as shown in yellow in these slices, because the $3 \mathrm{D}$ model is more stable and therefore, very small objects presented in a few slices may be ignored, such as blood vessels and internal lung airways.

The last analysis is the global analysis that reflects the same observations of the previous analysis: the proposed method obtained an average F-measure of $99.22 \% \pm 0.14,3 \mathrm{D}$ RG of $97.59 \% \pm 3.67$, EUOT of $97.39 \% \pm 4.35$, and LSCPM of $97.22 \% \pm 5.43$. Thus, one can say that the current methods under comparison attained average $\mathrm{F}$-measure values higher that $98.5 \%$ in the 


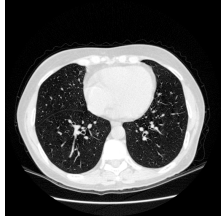

(a)

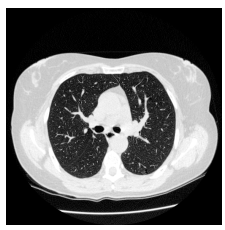

(f)

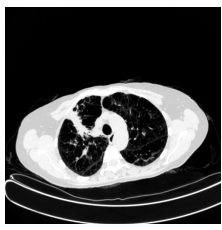

(k)

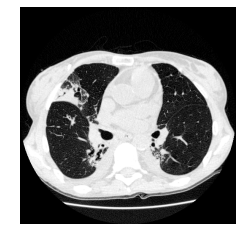

(p)

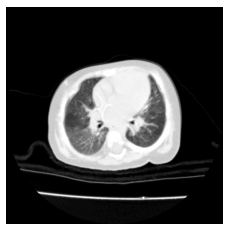

(u)

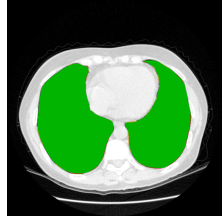

(b)

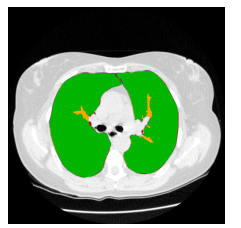

(g)

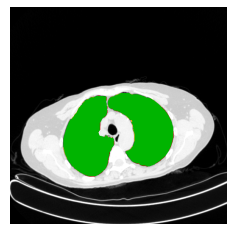

(1)

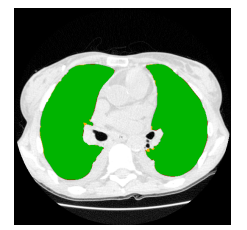

(q)

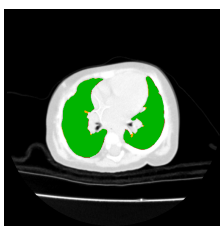

(v)

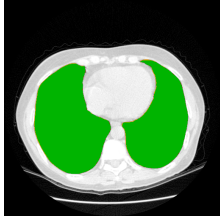

(c)

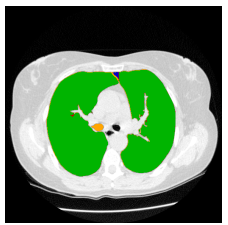

(h)

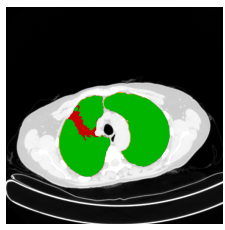

(m)

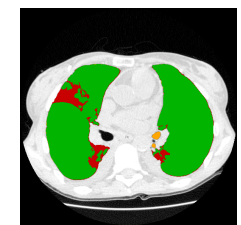

(r)

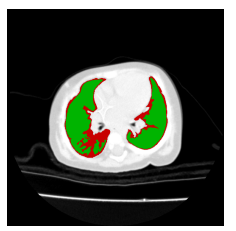

(w)

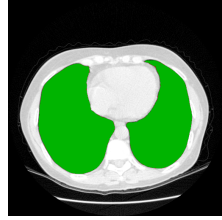

(d)

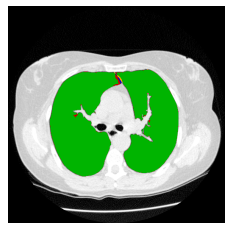

(i)

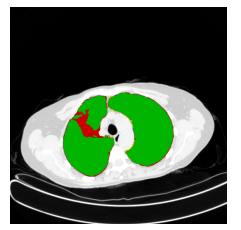

(n)

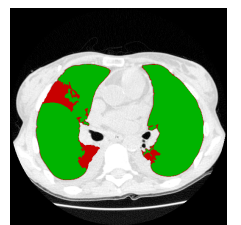

(s)

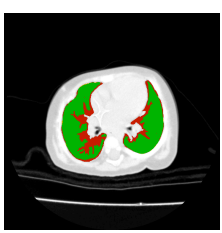

(x)

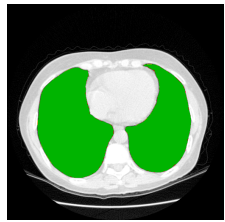

(e)

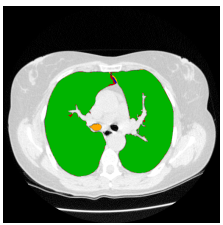

(j)

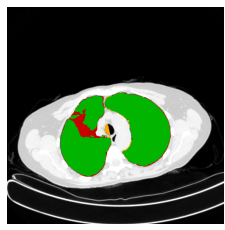

(o)

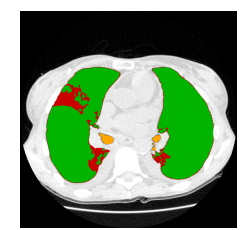

(t)

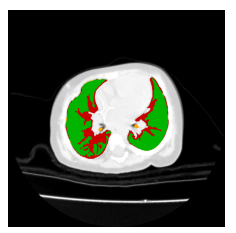

(y)

Figure 14: Examples of lung segmentations in CT scans obtained by the different methods under evaluation: a), f), k), p), u) original images; b), g), l), q) and v) 3D Adaptive Crisp ACM; c), h), m), r) and w) 3D Region Growing; d), i), n), s) and x) Level-set algorithm based on the coherent propagation method; and e), j), o), t) and y) semiautomatic segmentation by expert using the 3D OsiriX toolbox. 
healthy patients and in the patients with diseases that tend to diminish the HU density in some lung regions. However, the proposed method was more stable and obtained an average $\mathrm{F}$-measure over $99 \%$ in all tests independently of the type of disease presented.

Regarding the segmentation time, the most efficient methods in ascending order were the Level-set algorithm based on the coherent propagation method with $1.03 \pm 0.19$ minutes, 3D RG with $2.20 \pm 0.40$ minutes, 3D ACACM with $3.11 \pm 0.57$ minutes, and the semi-automatic segmentation by an expert using the $3 \mathrm{D}$ OsiriX toolbox with $3.89 \pm 0.66$ minutes. The proposed method obtained an average result of 3 minutes, which is three times the time required by the automatic commercial plugin software used to build the ground truth and 1.5 times of the $3 \mathrm{D}$ RG time. However, the 3D ACACM is eight times faster than the expert, which took 25 minutes on average in using the semiautomated commercial software with subsequent manual improvements.

The experimental dataset used and the results obtained are available at http://lapisco.ifce.edu.br/?page_id=131.

\section{Conclusion}

This work proposes a method called 3D Adaptive Crisp that is a new technique of automatic segmentation of lungs in CT scans of the thorax. The main contributions achieved by the proposed method are related to the new 3D Adaptive Crisp external energy, the novel 3D Adaptive Balloon internal energy and the robust 3D automatic initialization.

As secondary contributions, but also important to the quality of the results obtained, are the developed solutions for the addition, removal and initialization of points, which were not successfully overcome by previous studies. The use of the Sobel 3D operator allows better analysis of the objects present in the input image dataset through the proposed external energy. These contributions give a parametric method of active contour, such as Snakes, the ability to have results similar to the ones obtained by geometrical methods of active contours, such as Level Set, even when compared against an optimized Level-Set algorithm.

The 3D Adaptive Crisp was compared against three methods commonly used by specialists in the segmentation of CT scans of the thorax both from healthy and diseased patients, using a ground truth built by a medical expert.

The proposed method was comparatively more stable than the other methods independently of the diseased presented, obtaining an average F- 
measure over $99 \%$ in all tests. The findings confirmed that the proposed method is superior to the other methods under comparison, and its suitability to be used in clinical routine diagnosis, since it requires less than 4 minutes to accomplish the segmentation in a common personal computer.

As to future works, we intend to apply other computational intelligence and pattern recognition techniques to identify the origin of edges found in the lungs, to adapt the methods developed for the detection of other organs, and to investigate methods for the recognition of lung or other organ diseases.

\section{Acknowledgements}

Pedro P. Rebouças Filho acknowledges the sponsorship from the Federal Institute of Education, Science and Technology of Ceará, in Brazil, via Grants PROINFRA-IFCE/2013 and PROAPP-IFCE/2014. He also acknowledges the sponsorship from the Brazilian National Council for Research and Development (CNPq) through Grant 232644/2014-4.

Victor Hugo C. de Albuquerque acknowledges the sponsorship from the Brazilian National Council for Research and Development (CNPq) via Grants 470501/2013-8 and 301928/2014-2.

João Manuel R. S. Tavares thanks the funding of Project NORTE-010145-FEDER-000022 - SciTech - Science and Technology for Competitive and Sustainable Industries, co-financed by "Programa Operacional Regional do Norte" (NORTE2020), through "Fundo Europeu de Desenvolvimento Regional" (FEDER).

\section{References}

Al-Dossary, S., Al-Garni, K., 2013. New parametric 3d snake for medical segmentation of structures with cylindrical topology, in: SPE Saudi Arabia Section Technical Symposium and Exhibition, pp. 276-280.

Ariani, A., Carotti, M., Gutierrez, M., Bichisecchi, E., Grassi, W., Giuseppetti, G., Salaffi, F., 2014. Utility of an open-source dicom viewer software (OsiriX) to assess pulmonary fibrosis in systemic sclerosis: preliminary results. Rheumatology International 34, 511-516.

Arunkumar, R., 2012. Quantitative analysis of bronchiectasis using local binary pattern and fuzzy based spatial proximity, in: Recent Trends In 
Information Technology (ICRTIT), 2012 International Conference on, pp. $72-76$.

Astle, D., Hawkins, K., 2009. Begnning OpenGl Game Programming. Thomson, EUA. 2nd edition.

Berg, G., Julian, W., Mines, R., Richman, F., 1975. The constructive jordan curve theorem. Journal of Mathematics 5, 225-236.

Born, S., Dirkiwamaru, Pfeile, M., Bartz, D., 2009. 3-step segmentation of the lower airways with advanced leakage-control. IJCAI-09 workshop on Explanation-aware Computing (ExaCt 2009) , 239-255.

Campos, H., Lemos, A., 2009. Asthma and copd in view of the pulmonologist. Brazilian Journal of Pulmonology 35, 301-309.

Canas, S., Leehan, J., Jimenez-Alaniz, J., 2007. Plugin for OsiriX: Mean shift segmentation, in: Engineering in Medicine and Biology Society, 2007. EMBS 2007. 29th Annual International Conference of the IEEE, pp. 30603063.

De Nunzio, G., Tommasi, E., Agrusti, A., Cataldo, R., De Mitri, I., Favetta, M., Maglio, S., Massafra, A., Quarta, M., Torsello, M., Zecca, I., Bellotti, R., Tangaro, S., Calvini, P., Camarlinghi, N., Falaschi, F., Cerello, P., Oliva, P., 2011. Automatic lung segmentation in ct images with accurate handling of the hilar region. Journal of Digital Imaging 24, 11-27.

Diciotti, S., Lombardo, S., Falchini, M., Picozzi, G., Mascalchi, M., 2011. Automated segmentation refinement of small lung nodules in CT scans by local shape analysis. IEEE Transactions on Biomedical Engineering 58, 3418-3428.

Ge, Q., Shen, F., Jing, X.Y., Wu, F., Xie, S.P., Yue, D., Li, H.B., 2016. Active contour evolved by joint probability classification on riemannian manifold. Signal, Image and Video Processing , 1-8.

Hame, Y., Angelini, E., Hoffman, E., Barr, R., Laine, A., 2014. Adaptive quantification and longitudinal analysis of pulmonary emphysema with a hidden Markov measure field model. IEEE Transactions on Medical Imaging 33, 1527-1540. 
Haykin, S., 1999. Neural Networks: A Comprehensive Foundation. PrenticeHall, EUA. 2nd edition.

Irving, B., TaylorR, P., Todd-Pokropek, A., 2009. 3D segmentation of the airway tree using a morphology based method. IJCAI-09 workshop on Explanation-aware Computing (ExaCt 2009) , 297-307.

Juen, J., Cheng, Q., Schatz, B., 2015. A natural walking monitor for pulmonary patients using mobile phones. IEEE Journal of Biomedical and Health Informatics 19, 1399-1405.

Korfiatis, P., Kalogeropoulou, C., Karahaliou, A., Kazantzi, A., Costaridou, L., 2011. Vessel tree segmentation in presence of interstitial lung disease in MDCT. Information Technology in Biomedicine, IEEE Transactions on $15,214-220$.

Kwan, A., Fung, A., Jansen, P., Schivo, M., Kenyon, N., Delplanque, J.P., Davis, C., 2015. Personal lung function monitoring devices for asthma patients. IEEE Sensors Journal 15, 2238-2247.

Leninisha, S., Vani, K., 2015. Water flow based geometric active deformable model for road network. ISPRS Journal of Photogrammetry and Remote Sensing 102, $140-147$.

Lo, P., van Ginneken, B., Reinhardt, J., Yavarna, T., Jong, P., Irving, B., Fetita, C., Ortner, M., Pinho, R., Sijbers, J., Feuerstein, M., Fabijanska, A., Bauer, C., Beichel, R., Mendoza, C., Wiemker, R., Lee, J., Reeves, A., Born, S., Weinheimer, O., van Rikxoort, E., Tschirren, J., Mori, K., Odry, B., Naidich, D., Hartmann, I., Hoffman, E., Prokop, M., Pedersen, J., Bruijne, M., 2012. Extraction of airways from CT (EXACT'09). IEEE Transactions on Medical Imaging 31, 2093-2107.

Mansoor, A., Bagci, U., Xu, Z., Foster, B., Olivier, K., Elinoff, J., Suffredini, A., Udupa, J., Mollura, D., 2014. A generic approach to pathological lung segmentation. IEEE Transactions on Medical Imaging 33, 2293-2310.

Marco, R., Accordini, S., Cerveri, I., Corsico, A., Sunyer, J., Neukirch, F., Kunzly, N., Leynaert, B., Janson, C., Gislason, T., Vermeire, P., Svanes, C., Anto, J., Burney, P., 2004. An international survey of chronic obstrutive pulmonary disease in young adults according to gold stages. Thorax $59,120-125$. 
Martin, C.M., Roach, V.A., Nguyen, N., Rice, C.L., Wilson, T.D., 2013. Comparison of $3 \mathrm{~d}$ reconstructive technologies used for morphometric research and the translation of knowledge using a decision matrix. Anatomical Sciences Education 6, 393-403.

Matsuoka, S., Yamashiro, T., Washko, G., Kurihara, Y., 2010. Quantitative CT assessment of chronic obstructive pulmonary disease. RadioGraphics $30,55-66$.

Mesejo, P., Valsecchi, A., Marrakchi-Kacem, L., Cagnoni, S., Damas, S., 2015. Biomedical image segmentation using geometric deformable models and metaheuristics. Computerized Medical Imaging and Graphics 43, 167 $-178$.

Michael P Chae, David J Hunter-Smith, A.R.R.T.S.W.M.R., 2015. 3d volumetric analysis and haptic modeling for preoperative planning in breast reconstruction. Anaplastology $4,-$.

Mieloszyk, R., Verghese, G., Deitch, K., Cooney, B., Khalid, A., MirreGonzalez, M., Heldt, T., Krauss, B., 2014. Automated quantitative analysis of capnogram shape for copd normal and copd chf classification. IEEE Transactions on Biomedical Engineering 61, 2882-2890.

Moallem, P., Tahvilian, H., Monadjemi, S.A., 2015. Parametric active contour model using gabor balloon energy for texture segmentation. Signal, Image and Video Processing 10, 351-358.

Moreira, F.D.L., Kleinberg, M.N., Arruda, H.F., Freitas, F.N.C., Parente, M.M.V., de Albuquerque, V.H.C., Filho, P.P.R., 2016. A novel vickers hardness measurement technique based on adaptive balloon active contour method. Expert Systems with Applications 45, 294 - 306.

Nemec, S., Molinari, F., Dufresne, V., Gosset, N., Silva, M., Bankier, A., 2015. Comparison of four software packages for CT lung volumetry in healthy individuals. European Radiology 25, 1588-1597.

Presti, G., Carbone, M., Ciriaci, D., Aramini, D., Ferrari, M., Ferrari, V., 2015. Assessment of dicom viewers capable of loading patient-specific $3 \mathrm{~d}$ models obtained by different segmentation platforms in the operating room. Journal of Digital Imaging 28, 518-527. 
Pu, J., Fuhrman, C., Good, W., Sciurba, F., Gur, D., 2011. A differential geometric approach to automated segmentation of human airway tree. IEEE Transactions on Medical Imaging 30, 266-278.

Qiu, W., Yuan, J., Rajchl, M., Kishimoto, J., Chen, Y., de Ribaupierre, S., Chiu, B., Fenster, A., 2015. 3d MR ventricle segmentation in pre-term infants with post-hemorrhagic ventricle dilatation (PHVD) using multiphase geodesic level-sets. NeuroImage 118, $13-25$.

Ramalho, G.L.B., Rebouças Filho, P.P., Medeiros, F.N.S., Cortez, P.C., 2014. Lung disease detection using feature extraction and extreme learning machine. Research on Biomedical Engineering 30, 207 - 214.

Rebouças, E.S., Braga, A.M., Marques, R.C., Filho, P.P.R., 2016. A new approach to calculate the nodule density of ductile cast iron graphite using a level set. Measurement 89, $316-321$.

Rebouças Filho, P.P., Cortez, P.C., Felix, J.H.S., T. S. Cavalcante, T.d.S., Holanda, M.A., 2013. Adaptive 2D crisp active contour model applied to lung segmentation in CT images of the thorax of healthy volunteers and patients with pulmonary emphysema,. Research on Biomedical Engineering 29, 363-376.

Rebouças Filho, P.P., Cortez, P.C., Holanda, M.A., 2011. Active contour modes crisp: new technique for segmentation the lungs in CT images. Research on Biomedical Engineering 27, 259-272.

Rebouças Filho, P.P., Cortez, P.C., da Silva Barros, A.C., Albuquerque, V.H.C., 2014. Novel adaptive balloon active contour method based on internal force for image segmentation a systematic evaluation on synthetic and real images. Expert Systems with Applications 41, 7707 - 7721.

Schmitter, D., Gaudet-Blavignac, C., Piccini, D., Unser, M., 2015. Fault detection and characterization using a $3 \mathrm{~d}$ multidirectional sobel filter, in: 2015 IEEE International Conference on Image Processing (ICIP), pp. 276280.

Sellers, G., Junior, R.W., Haemel, N., 2015. OpenGL Superbible: Comprehensive tutorial and reference. Addison-Wesley, EUA. 7th edition. 
Sorensen, L., Nielsen, M., Lo, P., Ashraf, H., Pedersen, J., Bruijne, M., 2012. Texture-based analysis of copd: A data-driven approach. IEEE Transactions on Medical Imaging 31, 70-78.

Spina, G., Casale, P., Albert, P., Alison, J., Garcia-Aymerich, J., Costello, R., Hernandes, N., van Gestel, A., Leuppi, J., Mesquita, R., Singh, S., Smeenk, F., Tal-Singer, R., Wouters, E., Spruit, M., den Brinker, A., 2015. Identifying physical activity profiles in copd patients using topic models. IEEE Journal of Biomedical and Health Informatics 19, 1567-1576.

Sun, S., Bauer, C., Beichel, R., 2012. Automated 3-d segmentation of lungs with lung cancer in $\mathrm{CT}$ data using a novel robust active shape model approach. IEEE Transactions on Medical Imaging 31, 449-460.

Tschirren, J., Yavarna, T., Reinhardt, J., 2009. 3D segmentation of the airway tree using a morphology based method. IJCAI-09 workshop on Explanation-aware Computing (ExaCt 2009) , 227-238.

Van Rikxoort, E., Prokop, M., Hoop, B., Viergever, M., Pluim, J., van Ginneken, B., 2010. Automatic segmentation of pulmonary lobes robust against incomplete fissures. IEEE Transactions on Medical Imaging 29, $1286-1296$.

Wang, C., Frimmel, H., Smedby, ., 2011. Level-set based vessel segmentation accelerated with periodic monotonic speed function.

Wang, C., Frimmel, H., Smedby, ., 2014. Fast level-set based image segmentation using coherent propagation. Medical Physics 41.

Wei, J., Li, G., 2014. Automated lung segmentation and image quality assessment for clinical 3-D/4-D-computed tomography. Translational Engineering in Health and Medicine, IEEE Journal of 2, 1-10.

WHO, 2014. Global Strategy for the Diagnosis, Management and Prevention Chronic Obstrutive Pulmonar Disease. Technical Report. World Health Organization.

Wink, A.E., 2014. Pubic symphyseal age estimation from three-dimensional reconstructions of pelvic ct scans of live individuals. Journal of Forensic Sciences 59, 696-702. 
Wisniewski, M., Zielinski, T., 2015. Joint application of audio spectral envelope and tonality index in an e-asthma monitoring system. IEEE Journal of Biomedical and Health Informatics 19, 1009-1018. 